Article

\title{
An Integrated Study of the Serpentinite-Hosted Hydrothermal System in the Pollino Massif (Southern Apennines, Italy)
}

\author{
Giovanna Rizzo ${ }^{1}\left(\right.$, , Maria Carmela Dichicco ${ }^{1}$, Pedro Castiñeiras ${ }^{2}\left(\right.$ ) Fausto Grassa $^{3}(\mathbb{D}$, \\ Salvatore Laurita ${ }^{1}$, Michele Paternoster ${ }^{1,3}{ }^{-}$, Rosa Sinisi ${ }^{1,4, *}$ and Giovanni Mongelli ${ }^{1,4}$ \\ 1 Department of Sciences, University of Basilicata, 85100 Potenza, Italy; giovanna.rizzo@unibas.it (G.R.); \\ mc.dichicco@libero.it (M.C.D.); salvatorelaurita@hotmail.it (S.L.); michele.paternoster@unibas.it (M.P.); \\ giovanni.mongelli@unibas.it (G.M.) \\ 2 Departamento de Petrología y Geoquímica, Universidad Complutense, 28040 Madrid, Spain; \\ castigar@ucm.es \\ 3 Istituto Nazionale di Geofisica e Vulcanologia, Sezione di Palermo, 90146 Palermo, Italy; \\ fausto.grassa@ingv.it \\ 4 Istituto di Metodologie per l'Analisi Ambientale, Consiglio Nazionale delle Ricerche, \\ 85050 Tito Scalo (PZ), Italy \\ * Correspondence: rosa.sinisi@unibas.it or rosa.sinisi@imaa.cnr.it
}

Received: 18 November 2019; Accepted: 29 January 2020; Published: 31 January 2020

check for updates

\begin{abstract}
A comprehensive study of the serpentinite and associated veins belonging to the Frido Unit in the Pollino Massif (southern Italy) is presented here with the aim to provide new constraints about the hydrothermal system hosted by the accretionary wedge of the southern Apennines. The studied serpentinites are from two different sites: Fosso Arcangelo and Pietrapica. In both sites, the rocks show mylonitic-cataclastic structures and pseudomorphic and patch textures and are traversing by pervasive carbonate and quartz-carbonate veins. The mineralogical assemblage of serpentinites consists of serpentine group minerals (with a predominance of lizardite), amphiboles, pyroxene, chlorite, titanite, magnetite, and talc. In some samples, hydro-garnet was also detected and documented here for the first time. As for cutting veins, different mineralogical compositions were observed in the two sites: calcite characterizes the veins from Fosso Arcangelo, whereas quartz and dolomite are the principal minerals of the Pietrapica veins infill, suggesting a different composition of mineralizing fluids. Stable isotopes of $\mathrm{C}$ and $\mathrm{O}$ also indicate such a different chemistry. In detail, samples from the Pietrapica site are characterized by $\delta^{13} \mathrm{C}$ fluctuations coupled with a $\delta^{18} \mathrm{O}$ shift documenting calcite formation in an open-system where mixing between deep and shallow fluids occurred. Conversely, $\delta^{13} \mathrm{C}$ and $\delta^{18} \mathrm{O}$ of the Fosso Arcangelo veins show a decarbonation trend, suggesting their developing in a closed-system at deeper crustal conditions. Precipitation temperature calculated for both sites indicates a similar range $\left(80^{\circ} \mathrm{C}\right.$ to $\left.120^{\circ} \mathrm{C}\right)$, thus suggesting carbonate precipitation within the same thermal system.
\end{abstract}

Keywords: veins; hydrothermal fluids; C and O stable isotopes; Frido Units; southern Apennines

\section{Introduction}

Mantle peridotites are exposed on the seafloor at slow and ultraslow spreading mid-ocean ridges [1]. Seafloor spreading developed in areas characterized by tectonic extension and detachment faults, allowing uplift and exposure of mantle peridotite in oceanic core complexes [2-7]. Due to the interaction with seawater and deep hydrothermal fluids, seafloor peridotites are readily serpentinized over a wide range of conditions [8]. Serpentinites occurring in ophiolitic complexes are important for 
volatile cycling and fluid mobile elements acting as a source for water, carbon, sulfur, chlorine, boron, arsenic, and nitrogen [9-26]. These rocks, in fact, in the last years have been widely studied to gain a better understanding of their reactivity with respect to hydrothermal fluids promoting carbonation processes and mineral immobilization of large amounts of $\mathrm{CO}_{2}[27,28]$.

Carbonates, usually as serpentinite matrix and/or vein infill within an extensive network, are a common feature of altered oceanic lithosphere, although ocean-floor metasomatism is not the only process responsible for their presence in these rocks. Carbonates are common minerals that form in a number of geological settings and upon different environmental conditions. As the other chemically-derived precipitates, carbonate composition mirrors the physico-chemical properties and composition of the mineralizing fluid, a feature that helps to constrain the source fluid and environmental conditions that promote carbonate precipitation also under a complex tectonic scenario [29-36].

In southern Apennines, serpentinites occur as slices within the geological and structural frame of the Pollino Massif ophiolites (southern Ligurian Tethys) in the Ligurian Accretionary Complex (LAC) [37]. These rocks are traversed by a complex network of veins with different textural and macroscopic features. Their chemical and mineralogical compositions have never been studied before, although could better constrain the geological evolution of the serpentinites in the LAC of the Frido Unit.

With this in mind, we present here, for the first time, a comprehensive study of the serpentinites and associated carbonate and quartz-carbonate veins from the Pollino Massif, based on field observations, petrographic and mineralogical data, and carbon $\left(\delta^{13} \mathrm{C}_{\mathrm{V}-\mathrm{PDB}}\right)$ and oxygen $\left(\delta^{18} \mathrm{O}_{\mathrm{V}-\mathrm{SMOW}}\right)$ stable isotopes, to provide new constraints about the hydrothermal system hosted by the accretionary wedge of southern Apennines. Our principal goals are to define (1) source and composition of mineralizing fluids and (2) processes leading to mobilization, fractionation, and redistribution of chemical elements during the emplacement of the Frido Unit serpentinites within the accretionary wedge.

\section{Geological Background}

The Pollino Massif is located in the southern Apennines at the Calabria-Lucania border zone (Figure 1). It consists of tectonically juxtaposed thrust belts derived from the deformation of the African passive margin [38]. The deformation occurred between Oligocene and Pleistocene and involved the Ligurian ocean ophiolitic crust and its sedimentary cover [38,39]. In this area, the Liguride Complex [38], also defined as Liguride Units [40], is well exposed and is located in the highest position in the tectonics edifice of the southern Apennines. The Liguride Complex is derived from the NW subduction of the Tethyan ocean-continent transition zone and was divided into different tectonic units, where fragments of Jurassic oceanic crust [38], associated with slices of continental crust rocks, are preserved $[38,39,41,42]$. These terrains consist of a Mesozoic to Cenozoic flysch and a series of ophiolitic nappes, widely exposed along the whole Apennine Chain and in Calabria [38].

The Liguride Complex has been subdivided into two units, the metamorphosed Frido Unit and the non-metamorphic Calabro-Lucano Flysch [39] or North Calabria Unit [40]. The Frido Unit forms the uppermost thrust sheet, and tectonically overlies the North Calabrian Units, which in turn is split in a number of thrust sheets [39].

The Frido Unit consists of a metasedimentary sequence (phyllite, meta-arenite, quartzite, and isolated bodies of meta-limestone or calcschist and metapelite) [37,43-46] with blocks of both oceanic and continental rocks [38,41,42]. Oceanic lithosphere in the Frido Unit [47] is represented by tectonized serpentinite [48-51], metabasalt [52], metagabbro, metapillow lavas [37], and dismembered metadoleritic dykes [53-56]. Continental crust rocks mainly consist of weathered granofels, garnet gneiss, garnet-biotite gneiss, leucocratic biotite gneiss and lenticular bodies of amphibolite [41,42] often cross-cut by basic dykes [41].

According to several studies $[38,39,41,53,54,57,58]$, the Frido Unit underwent a polyphase blueschist to greenschist facies metamorphism developed in the deeper parts of the Liguride accretionary wedge. The blueschist metamorphism in mafic rocks developed at peak pressure conditions of 
0.6-0.8 GPa and temperatures of $350{ }^{\circ} \mathrm{C}$, whereas the subsequent greenschist facies overprint took place at $P=0.4 \mathrm{GPa}$ and $T=300-350{ }^{\circ} \mathrm{C}[37,59]$. Cavalcante et al. [60] interpreted that also the nearby metasediments were affected by HP-LT conditions using illite crystallinity data and the b0 parameter of K-white mica in phyllite. Similar metamorphic conditions have also been documented by Invernizzi et al. [61] and Laurita and Rizzo [62] for the metabasites of the Frido Unit $\left(200-300{ }^{\circ} \mathrm{C}\right.$ and 0.6-0.8 GPa; 300-400 ${ }^{\circ} \mathrm{C}$ and 0.8-1.2 GPa, respectively).

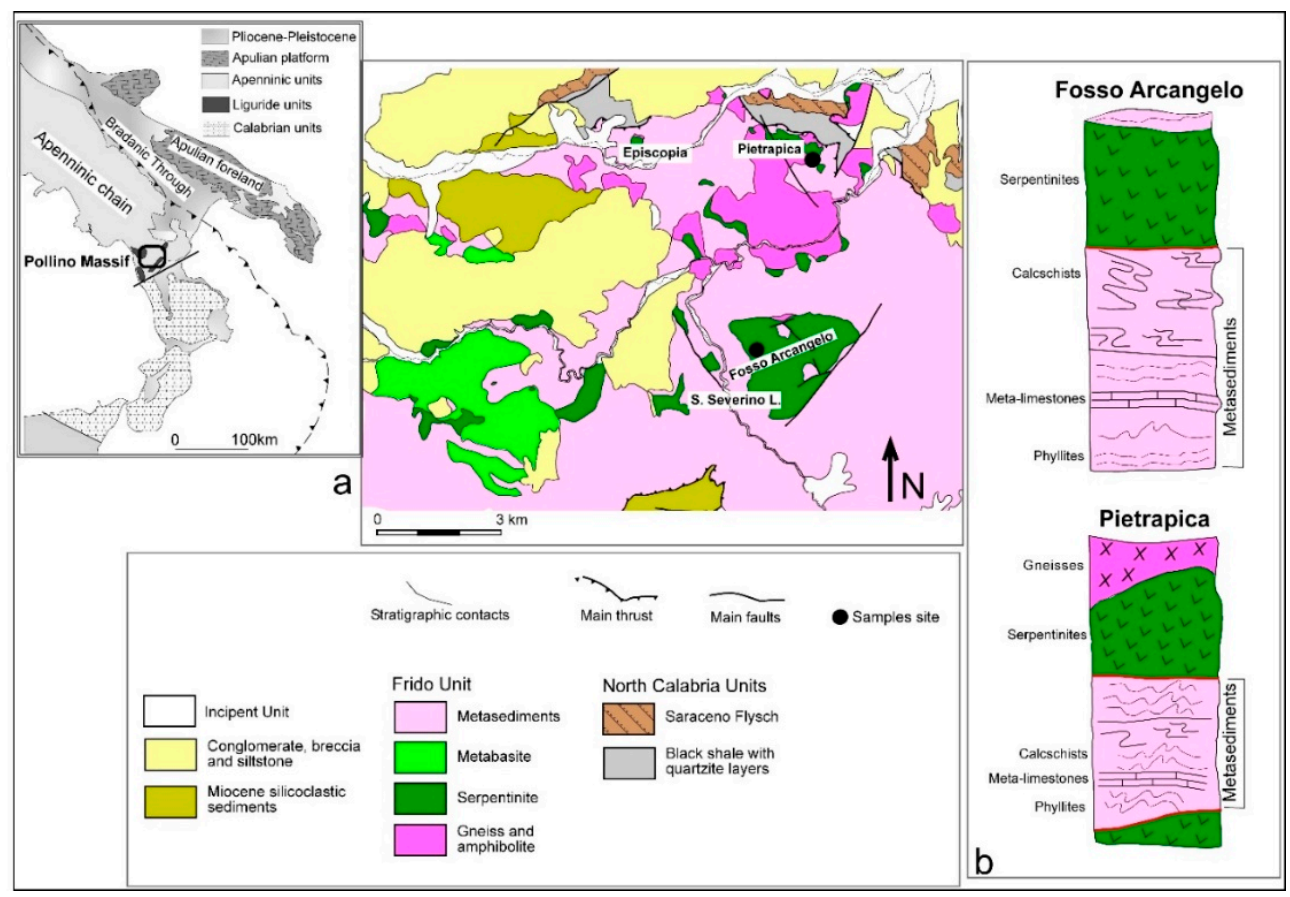

Figure 1. (a) Synthetic geological map of the Liguride Complex in the Pollino Massif and location of the study area, (b) tectonic and stratigraphic sequence with the ultramaphic and continental rocks contact (not to scale).

During blueschist facies conditions, crystallization of glaucophane occurred in metabasites [63], and of magnesio-riebeckite in schist associated with metabasites [64] and carpholite in some veins in metapelites and phyllites [37,65]. Afterward, in a brittle deformation regime, the most obvious structures are characterized by cataclastic bands well developed in the serpentinites [63].

\section{Sampling and Analytical Method}

A total of 26 representative samples of serpentinites dominated by carbonate-veins (SpFA) and serpentinites dominated by quartz-carbonate veins (SpPP) were collected at the Fosso Arcangelo site, near the San Severino Lucano village, and in the Pietrapica site, at the Calabria-Lucanian boundary (Figure 1) (Table 1).

The serpentinites outcropping at the Fosso Arcangelo site (SpFA) are brownish-grey-green and appear intensely reworked by strong brittle and ductile deformation, evidenced by several slip surfaces and by the presence of isoclinal and tight folds and some intrafolial folds often associated with crenulation cleavage. The fractures are commonly filled by carbonate minerals and display changes in both thickness and length (Figure 2a).

Serpentinites in this site crosscut by metadolerite dykes that were affected by metamorphism under relatively HP/LT (blueschist facies) conditions during the formation of the Apennine accretionary prism [45,53-55]. The Pietrapica site is characterized by a complex exposure of dark-green cataclastic serpentinites (SpPP) with several slip surfaces and badland-like morphology. The serpentinite shows quartz-carbonate veins with talc-rich domains (Figure 2b). At the surrounding of the Pietrapica area, no rocks with any experience of HP/LT metamorphism occur. 
Table 1. GPS coordinates, texture, and mineral assemblages of the serpentinite samples.

\begin{tabular}{|c|c|c|c|c|}
\hline $\begin{array}{l}\text { Sample } \\
\text { No. }\end{array}$ & $\begin{array}{l}\text { Sample } \\
\text { Code }\end{array}$ & $\begin{array}{c}\text { GPS } \\
\text { Coordinates }\end{array}$ & Texture & Mineral Assemblage \\
\hline \multicolumn{5}{|c|}{ Samples from the Fosso Arcangelo site (SpFA) } \\
\hline 1 & SpFA2 & $\begin{array}{l}41^{\circ} 01^{\prime} 40.2^{\prime \prime} \mathrm{N} \\
16^{\circ} 08^{\prime} 09.6^{\prime \prime} \mathrm{E}\end{array}$ & Breccia, pseudomorphic and veins texture & $\begin{array}{c}\text { Srp-Cal-Am-Pren-Chl-Mag } \\
\text { and Ol-Opx relict }\end{array}$ \\
\hline 2 & SpFA5 & $\begin{array}{l}40^{\circ} 01^{\prime} 39.2^{\prime \prime} \mathrm{N} \\
16^{\circ} 08^{\prime} 09.6^{\prime \prime} \mathrm{E}\end{array}$ & Breccia, veins texture & Srp-Cal-Am-Chl-Mag-Ttn \\
\hline 3 & SpFA6.3 & $\begin{array}{l}40^{\circ} 01^{\prime} 38.3^{\prime \prime} \mathrm{N} \\
16^{\circ} 08^{\prime} 10.4^{\prime \prime} \mathrm{E}\end{array}$ & $\begin{array}{l}\text { Breccia, pseudomorphic, veins and } \\
\text { patch texture }\end{array}$ & $\begin{array}{l}\text { Srp-Cal-Am-Chl-Mag-Pmp } \\
\text { and Ol-Opx-Cpx relict }\end{array}$ \\
\hline 4 & SpFA7 & $\begin{array}{l}40^{\circ} 01^{\prime} 37.6^{\prime \prime} \mathrm{N} \\
16^{\circ} 08^{\prime} 10.7^{\prime \prime} \mathrm{E}\end{array}$ & Pseudomorphyc and veins texture & $\begin{array}{l}\text { Srp-Cal-Am-Chl-Pmp-Ol- } \\
\text { Opx-Cpx relict-Mag }\end{array}$ \\
\hline 5 & SpFA7a & $\begin{array}{l}40^{\circ} 01^{\prime} 37.6^{\prime \prime} \mathrm{N} \\
16^{\circ} 08^{\prime} 10.7^{\prime \prime} \mathrm{E}\end{array}$ & Pseudomorphyc, veins and patch texture & $\begin{array}{l}\text { Srp-Cal-Am-Chl-Mag and } \\
\text { Ol-Opx-Cpx relict }\end{array}$ \\
\hline 6 & SpFA9 & $\begin{array}{l}40^{\circ} 01^{\prime} 37.0^{\prime \prime} \mathrm{N} \\
16^{\circ} 08^{\prime} 11.9^{\prime \prime} \mathrm{E}\end{array}$ & $\begin{array}{l}\text { Schistosity with crenulation cleavage, } \\
\text { veins texture }\end{array}$ & Srp-Cal-Di-Adr-Mag \\
\hline 7 & SpFA37 & $\begin{array}{l}40^{\circ} 01^{\prime} 37.0^{\prime \prime} \mathrm{N} \\
16^{\circ} 08^{\prime} 11.9^{\prime \prime} \mathrm{E}\end{array}$ & $\begin{array}{l}\text { Schistosity with crenulation cleavage, } \\
\text { veins texture and protomylonitic fabric }\end{array}$ & Srp-Cal-Di-Adr-Mag \\
\hline 8 & SpFA38 & $\begin{array}{l}40^{\circ} 01^{\prime} 37.0^{\prime \prime} \mathrm{N} \\
16^{\circ} 08^{\prime} 11.9^{\prime \prime} \mathrm{E}\end{array}$ & $\begin{array}{l}\text { Schistosity with crenulation cleavage, } \\
\text { veins texture and protomylonitic fabric }\end{array}$ & Srp-Cal-Di-Adr-Mag \\
\hline 9 & SpFA39 & $\begin{array}{l}40^{\circ} 01^{\prime} 37.0^{\prime \prime} \mathrm{N} \\
16^{\circ} 08^{\prime} 11.9^{\prime \prime} \mathrm{E}\end{array}$ & $\begin{array}{l}\text { Schistosity with crenulation cleavage, } \\
\text { veins texture and protomylonitic fabric }\end{array}$ & Srp-Cal-Di-Adr-Mag \\
\hline 10 & SpFA40 & $\begin{array}{l}40^{\circ} 01^{\prime} 37.0^{\prime \prime} \mathrm{N} \\
16^{\circ} 08^{\prime} 11.9^{\prime \prime} \mathrm{E}\end{array}$ & $\begin{array}{l}\text { Schistosity with crenulation cleavage, } \\
\text { veins texture and protomylonitic fabric }\end{array}$ & Srp-Cal-Di-Adr-Mag \\
\hline 11 & SpFA41 & $\begin{array}{l}40^{\circ} 01^{\prime} 37.0^{\prime \prime} \mathrm{N} \\
16^{\circ} 08^{\prime} 11.9^{\prime \prime} \mathrm{E}\end{array}$ & $\begin{array}{l}\text { Schistosity with crenulation cleavage and } \\
\text { veins texture }\end{array}$ & Srp-Cal-Di-Adr-Mag \\
\hline 12 & SpFA42 & $\begin{array}{l}40^{\circ} 01^{\prime} 37.0^{\prime \prime} \mathrm{N} \\
16^{\circ} 08^{\prime} 11.9^{\prime \prime} \mathrm{E}\end{array}$ & $\begin{array}{l}\text { Schistosity with crenulation cleavage, } \\
\text { veins texture and protomylonitic fabric }\end{array}$ & Srp-Cal-Di-Adr-Mag \\
\hline 13 & SpFA43 & $\begin{array}{l}40^{\circ} 01^{\prime} 37.0^{\prime \prime} \mathrm{N} \\
16^{\circ} 08^{\prime} 11.9^{\prime \prime} \mathrm{E}\end{array}$ & $\begin{array}{l}\text { Schistosity with crenulations cleavage } \\
\text { and veins texture }\end{array}$ & Srp-Cal-Di-Adr-Mag \\
\hline 14 & SpFA44 & $\begin{array}{l}40^{\circ} 01^{\prime} 37.0^{\prime \prime} \mathrm{N} \\
16^{\circ} 08^{\prime} 11.9^{\prime \prime} \mathrm{E}\end{array}$ & $\begin{array}{l}\text { Schistosity with crenulation cleavage, } \\
\text { veins texture and protomylonitic fabric }\end{array}$ & Srp-Cal-Di-Adr-Mag \\
\hline 15 & SpFA45 & $\begin{array}{l}40^{\circ} 01^{\prime} 37.0^{\prime \prime} \mathrm{N} \\
16^{\circ} 08^{\prime} 11.9^{\prime \prime} \mathrm{E}\end{array}$ & $\begin{array}{l}\text { Schistosity with crenulation cleavage, } \\
\text { veins texture and protomylonitic fabric }\end{array}$ & Srp-Cal-Di-Adr-Mag \\
\hline 16 & SpFA46 & $\begin{array}{l}40^{\circ} 01^{\prime} 37.0^{\prime \prime} \mathrm{N} \\
16^{\circ} 08^{\prime} 11.9^{\prime \prime} \mathrm{E}\end{array}$ & $\begin{array}{l}\text { Schistosity with crenulations cleavage } \\
\text { and veins texture }\end{array}$ & Srp-Cal-Di-Adr-Mag \\
\hline 17 & SpFA28 & $\begin{array}{l}40^{\circ} 01^{\prime} 45.3^{\prime \prime} \mathrm{N} \\
16^{\circ} 0.8^{\prime} 27.3^{\prime \prime} \mathrm{E}\end{array}$ & $\begin{array}{l}\text { Pseudomorphyc texture-veins texture } \\
\text { Patch texture }\end{array}$ & $\begin{array}{l}\text { Srp-Cal-Am-Chl-Mag and } \\
\text { Ol-Opx-Cpx relict }\end{array}$ \\
\hline 18 & SpFA29 & $\begin{array}{l}40^{\circ} 01^{\prime} 45.3^{\prime \prime} \mathrm{N} \\
16^{\circ} 0.8^{\prime} 27.3^{\prime \prime} \mathrm{E}\end{array}$ & $\begin{array}{l}\text { Pseudomorphyc texture Veins texture } \\
\text { Patch texture }\end{array}$ & $\begin{array}{l}\text { Srp-Cal-Am-Chl-Mag and } \\
\text { Ol-Opx-Cpx relict }\end{array}$ \\
\hline 19 & SpFA30 & $\begin{array}{l}40^{\circ} 01^{\prime} 45.3^{\prime \prime} \mathrm{N} \\
16^{\circ} 0.8^{\prime} 27.3^{\prime \prime} \mathrm{E}\end{array}$ & $\begin{array}{l}\text { Pseudomorphyc texture Veins texture } \\
\text { Patch texture }\end{array}$ & $\begin{array}{l}\text { Srp-Cal-Am-Chl-Mag and } \\
\text { Ol-Opx-Cpx relict }\end{array}$ \\
\hline 20 & SpFA10 & $\begin{array}{l}40^{\circ} 02^{\prime} 56,8^{\prime \prime} \mathrm{N} \\
16^{\circ} 09^{\prime} 01.6^{\prime \prime} \mathrm{E}\end{array}$ & $\begin{array}{l}\text { Pseudomorphyc texture Veins texture } \\
\text { Patch texture }\end{array}$ & $\begin{array}{l}\text { Srp-Cal-Am-Chl-Mag and } \\
\text { Ol-Opx-Cpx relict }\end{array}$ \\
\hline \multicolumn{5}{|c|}{ Samples from the Pietrapica quarry (SpPP) } \\
\hline 21 & SpPP31 & $\begin{array}{l}40^{\circ} 04^{\prime} 08.6^{\prime \prime} \mathrm{N} \\
16^{\circ} 0.9^{\prime} 19.6^{\prime \prime} \mathrm{E}\end{array}$ & Patch texture, veins texture & Srp-Cal \pm Am-Chl-Mag \\
\hline 22 & SpPP32 & $\begin{array}{l}40^{\circ} 04^{\prime} 08.6^{\prime \prime} \mathrm{N} \\
16^{\circ} 0.9^{\prime} 19.6^{\prime \prime} \mathrm{E}\end{array}$ & Brecciated textures & Tlc-Qtz-Cal \\
\hline 23 & SpPP33 & $\begin{array}{l}40^{\circ} 04^{\prime} 08.6^{\prime \prime} \mathrm{N} \\
16^{\circ} 0.9^{\prime} 19.6^{\prime \prime} \mathrm{E}\end{array}$ & Patch texture, veins texture & Srp-Cal \pm Am-Chl-Mag \\
\hline 24 & SpPP34 & $\begin{array}{l}40^{\circ} 04^{\prime} 08.6^{\prime \prime} \mathrm{N} \\
16^{\circ} 0.9^{\prime} 19.6^{\prime \prime} \mathrm{E}\end{array}$ & Brecciated textures & Tlc-Qtz-Cal \\
\hline 25 & SpPP35 & $\begin{array}{l}40^{\circ} 04^{\prime} 08.6^{\prime \prime} \mathrm{N} \\
16^{\circ} 0.9^{\prime} 19.6^{\prime \prime} \mathrm{E}\end{array}$ & Patch texture, veins texture & Srp-Cal \pm Am-Chl-Mag \\
\hline 26 & SpPP36 & $\begin{array}{l}40^{\circ} 04^{\prime} 08.6^{\prime \prime} \mathrm{N} \\
16^{\circ} 0.9^{\prime} 19.6^{\prime \prime} \mathrm{E}\end{array}$ & Brecciated textures & Tlc-Qtz-Cal \\
\hline
\end{tabular}

Petrographic characterization of all samples was carried out by optical microscopy on thin sections of rock samples oriented following their foliations and lineations. 

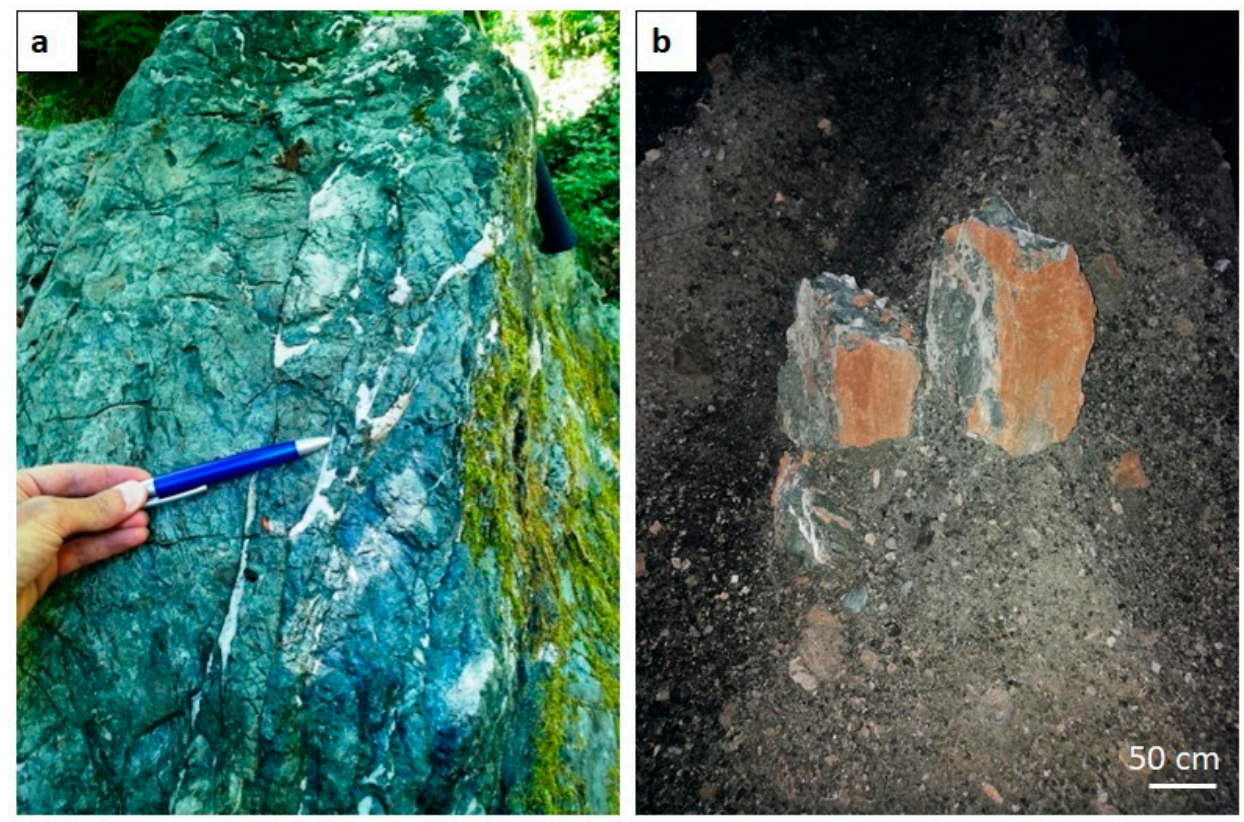

Figure 2. (a) Carbonate veins in the serpentinite from the Fosso Arcangelo site (SpFA); (b) Cataclastic serpentinites with quartz-carbonate veins and talc-rich domains in the Pietrapica quarry (SpPP).

Mineralogy analyses were performed on randomly oriented powdered samples of both host-rocks and vein infill by using X-ray powder diffraction (XRPD) and $\mu$-Raman techniques at the Department of Sciences, University of Basilicata (Potenza, Italy). XRPD analyses were accomplished by means of Siemens D5000 equipment with $\mathrm{CuK} \alpha$ radiation, $40 \mathrm{kV}$ and $32 \mathrm{~mA}, 2$ s per step, and a step scan of $0.02^{\circ} 2 \theta$. Data were recorded between 5 and $70^{\circ} 2 \theta$ for the bulk rock samples and from 15 to $70^{\circ} 2 \theta$ for the vein infill. The mineral phase identification was carried out by means of the X'Pert HighScore Plus software (PANalytical 2001, Version 01 using the PDF-2 (2005) database. The $\mu$-Raman analyses were carried out using a Horiba Jobin-Yvon LabRam HR800 spectrometer equipped with a HeNe laser source with a wavelength of $633 \mathrm{~nm}$, a CCD detector operating at $-70{ }^{\circ} \mathrm{C}$, and an edge filter that excludes from detection shift below $150 \mathrm{~cm}^{-1}$. A spectral resolution of $4 \mathrm{~cm}^{-1}$ was obtained by a holographic grating with 600 lines $/ \mathrm{mm}$. Correct calibration of the instrument was verified checking the position of the Si band at $\pm 520.7 \mathrm{~cm}^{-1}$. Output laser power was $20 \mathrm{mV}$, and measurements were performed using an optical microscope Olympus with objectives of $10 \times, 50 \times$, and $100 \times$. A laser beam spatial resolution of $1 \mu \mathrm{m}$ was obtained with the $100 \times$ objective. Spectra results were from the average of 5 acquisitions of $10 \mathrm{~s}$ to optimize the signal/noise ratio. Two regions of the Raman spectra were investigated: $1200-150 \mathrm{~cm}^{-1}$ for structural bonding characterization and $3800-3500 \mathrm{~cm}^{-1}$ for the characterization of the hydroxyl groups. The minerals were identified based on the data reported in the online RUFF database.

Mineral chemistry was determined at the Centro Nacional de Microscopía Electrónica (CNME) of the Universidad Complutense (Madrid, Spain) by electron microprobe (EMP) analyses on the serpentinites and associated veins, using a JEOL Superprobe JXA-8900M equipped with four wavelength dispersive spectrometers. Silicate and oxide analyses were conducted at an accelerating voltage of $15 \mathrm{kV}$, an electron beam current of $20 \mathrm{nA}$, and a beam diameter of $5 \mu \mathrm{m}$. An accelerating voltage of $20 \mathrm{kV}$, an electron beam current of $10 \mathrm{nA}$, and a beam diameter of $5 \mu \mathrm{m}$ were used for carbonate minerals. Each element was counted for $15 \mathrm{~s}$. The following minerals were used as standards: sillimanite for the $\mathrm{Al}$, albite for the $\mathrm{Si}$ and $\mathrm{Na}$, almandine for the $\mathrm{Mn}$ and $\mathrm{Fe}$, kaersutite for $\mathrm{Mg}$, $\mathrm{Ti}$ and $\mathrm{Ca}$, microcline for the $\mathrm{K}$, fluorapatite for the $\mathrm{P}, \mathrm{Ca}, \mathrm{F}, \mathrm{Cl}$, and $\mathrm{Ni}, \mathrm{Cr}$ pure metals. Corrections were made using the $\mathrm{ZAF}$ (Z: atomic number; A: absorption; F: fluorescence) method. The estimation uncertainties for major and minor elements were determined for each analysis, which have uncertainties from $\pm 0.8 \%$ to $\pm 5 \%$. The structural formula of amphiboles was recalculated on the basis of 23 oxygens and classified by using the amphiboles nomenclature suggested by Leake et al. [66,67]. The andradite structural formula 
was recalculated on the basis of 24 oxygens. The structural formula of pyroxene was recalculated on the basis of 6 oxygens.

A total of 21 vein infills were selected for stable oxygen and carbon isotope analysis including 15 samples from the SpFA and 6 samples from the SpPP. In detail, about $0.1 \mathrm{mg}$ of powder samples were put in a $12 \mathrm{~mL}$ screw cap Exetainer(R) vial and then flushed with pure helium to remove the air in the headspace. Subsequently, about $50 \mu \mathrm{L}$ of $100 \% \mathrm{H}_{3} \mathrm{PO}_{4}$ was added to each sample for the conversion to carbon dioxide. The analyses were performed using a Thermo GB-II peripheral coupled with a Thermo Delta V Plus CF-IRMS at "Istituto Nazionale di Geofisica e Vulcanologia-Sezione di Palermo (Italy) laboratories" (Palermo, Italy). The results are reported in delta per mil (\%o) values relative to the Vienna Pee Dee Belemnite (V-PDB) international scale. The $\delta^{18} \mathrm{O}$ values on the V-PDB scale are converted to the Vienna Standard Mean Ocean Water (V-SMOW) using the equation of Coplen et al. [68]:

$$
\delta^{18} \mathrm{O}_{\mathrm{V}-\mathrm{SMOW}}=1.03091 * \delta^{18} \mathrm{O}_{\mathrm{V}-\mathrm{PDB}}+30.91
$$

and are expressed in delta per mil (\%o) relative to the Vienna Standard Mean Ocean Water (V-SMOW) value. A Carrara Marble internal standard (MAB) and two international standards (NBS-18 and NBS-19) were used for calibration. Analytical reproducibility was better than $0.1 \%$ ond $0.15 \%$ o for $\delta^{13} \mathrm{C}$ and $\delta^{18} \mathrm{O}$, respectively.

Some quartz-carbonate veins of the SpPP were selected for the study of fluid inclusions and for the microthermometric determinations performed at the Departamento de Estratigrafía, Universidad Complutense, (Madrid, Spain). Doubly polished $100 \mu \mathrm{m}$-thick sections were prepared for the samples without any heating and glued to frosted glass with cyanoacrylate. The microthermometric study was performed on the selected portions of samples in a linkam THMSG-600 heating and freezing stage. The stage was calibrated with synthetic fluid inclusions, including the triple point of $\mathrm{CO}_{2}$, melting point of $\mathrm{H}_{2} \mathrm{O}$, and critical point of $\mathrm{H}_{2} \mathrm{O}$. The melting point of $\mathrm{H}_{2} \mathrm{O}$ standards show that the accuracy for low-temperature measurements is better than $\pm 0.1^{\circ} \mathrm{C}$. Critical point standards show that accuracy for high-temperature measurements is better $\pm 0.1^{\circ} \mathrm{C}$. Homogenization temperatures (Th) have been interpreted as minimum entrapment temperatures. In this case, no pressure corrections were applied because a pressure determination would involve too many error-prone assumptions without an independently obtained value of pressure. The interpretation of Th as minimum entrapment temperatures is a typical procedure in working with Th data $[69,70]$. To interpret salinity, a $\mathrm{NaCl}-\mathrm{H}_{2} \mathrm{O}$ model (using the equations from [71]) was used on the basis of the observed ice-melting temperatures of the last ice crystal $\mathrm{Tm}$ (Ice) from fluid inclusions.

\section{Results}

\subsection{Petrography}

\subsubsection{The SpFA}

The SpFA consist of serpentinite breccias with locally protomylonitic fabric and crosscutting carbonate veins. The serpentinite breccias (Figure 3a) are characterized by angular and irregularly shaped serpentinite grains embedded in the carbonate matrix. In the protomylonitic portion, breccias show differentiated crenulation cleavage with antisymmetric microfolds. The main foliation is defined by cleavage domains (limbs of microfolds) and microlithons (fold hinge areas). The main foliation is well-developed and is marked by hydro-andradite. This schistosity refolded the previous foliation (Figure $3 b$ ) defined by recrystallized serpentine, metamorphic clinopyroxene, and accessory minerals such as titanite and magnetite. 

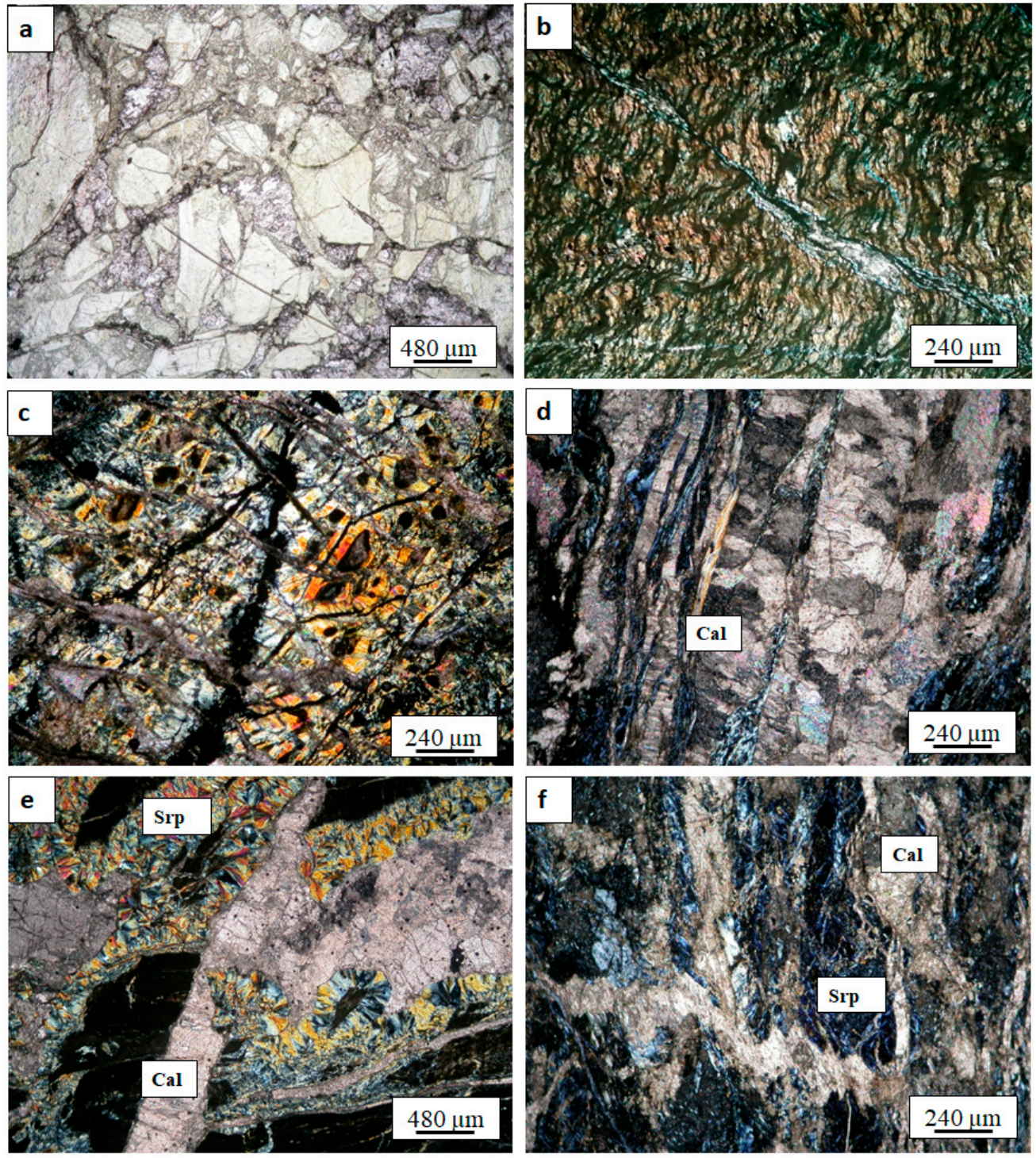

Figure 3. Photomicrographs of SpFA samples: (a) The serpentinite breccia characterized by serpentinite fragments in a carbonate matrix, plane polarized light, $4 \times$; (b) schistosity with crenulation cleavage, crossed polarized light, $4 \times$; (c) relict olivine grains in the core of the mesh-texture. Olivine is replaced by calcite, the mesh-texture are cross-cut by carbonate veins, crossed polarized light, $4 \times$; (d) sub-parallel carbonate veins, crossed polarized light, $4 \times$; (e) vein infill with carbonate and serpentine minerals, crossed polarized light, $4 \times$; (f) carbonate veins with anastomosing network, crossed polarized light, $4 \times$. $\mathrm{Cal}=$ calcite; $\operatorname{Srp}=$ serpentine. Abbreviation after [72].

The SpFA samples consist of a primary mineral assemblage made up of olivine, orthopyroxene and clinopyroxene (augite), and a serpentinite assemblage consisting of serpentine group minerals (mainly lizardite and a minor amount of chrysotile and antigorite), tremolite, diopside, and clinochlore. Accessory minerals are garnet, titanite, magnetite, and carbonate phases. As recently documented by Dichicco et al. [73], locally edenite amphibole may occur in chlorite-free samples.

Lizardite + magnetite mesh texture or hourglass structures [74-76] usually occur in the SpFA showing cores of relict olivine grains replaced by calcite that locally are cross-cut by carbonate veins (Figure 3c). Along cleavage planes, lamellae and fibers of lizardite and chrysotile are spread on bastite pseudomorphs after pyroxene.

Orthopyroxene is replaced by bastite pseudomorphs with exsolution lamellae of lizardite or fine-grained diopside aggregates. Primary magmatic clinopyroxene (augite) is preserved or replaced 
by metamorphic diopside. Spinel is replaced by $\mathrm{Cr}$ and Al-magnetite at the core and clinochlore at the rim.

Several types of carbonate veins have been recognized in the SpFA samples. As shown in Figure $3 \mathrm{~d}$,e, veins crosscut the bulk rock and are distinguished in sheeted (micrite-filled veins, carbonate veins with serpentine, carbonate veins with amphibole, fibrous calcite veins) and carbonate types displaying different thickness. Veins occasionally form an anastomosing network with acicular, fibrous, and radial serpentine and amphibole crystals (Figure 3f).

\subsubsection{The SpPP}

The SpPP are characterized by serpentinite breccias with quartz-carbonate rich veins. The serpentinite assemblage is made up of lizardite and minor chrysotile and antigorite, carbonate minerals, amphibole minerals (actinolite, tremolite), clinochlore, Cr-spinel, quartz, and talc. Magnetite is the only accessory mineral. Similarly to the SpFA, in the SpPP, lizardite occurs in the relict mesh texture and in the matrix.

An irregular patchy texture with carbonates growing after serpentine (Figure 4a) characterizes the SpPP samples. The carbonates occur as microcrystals, together with talc and rare fibrous tremolite. They occur as elongated rombohedric crystals (Figure 4b) only in the veins. Locally, the SpPP samples are characterized by talc-rich domains where quartz and carbonate minerals are also present. Quartz is in micrometer sub-grains, showing undulatory extinction and dynamic recrystallization [77] and intergrowths with carbonate crystals (Figure 4c). Talc, mostly associated with serpentine (lizardite and chrysotile) and chlorite, occurs as massive coarse- to medium-grained aggregates, fine fibers, and/or tabular crystals with perfect cleavage on the [001] plane (Figure $4 \mathrm{~d}$ ).
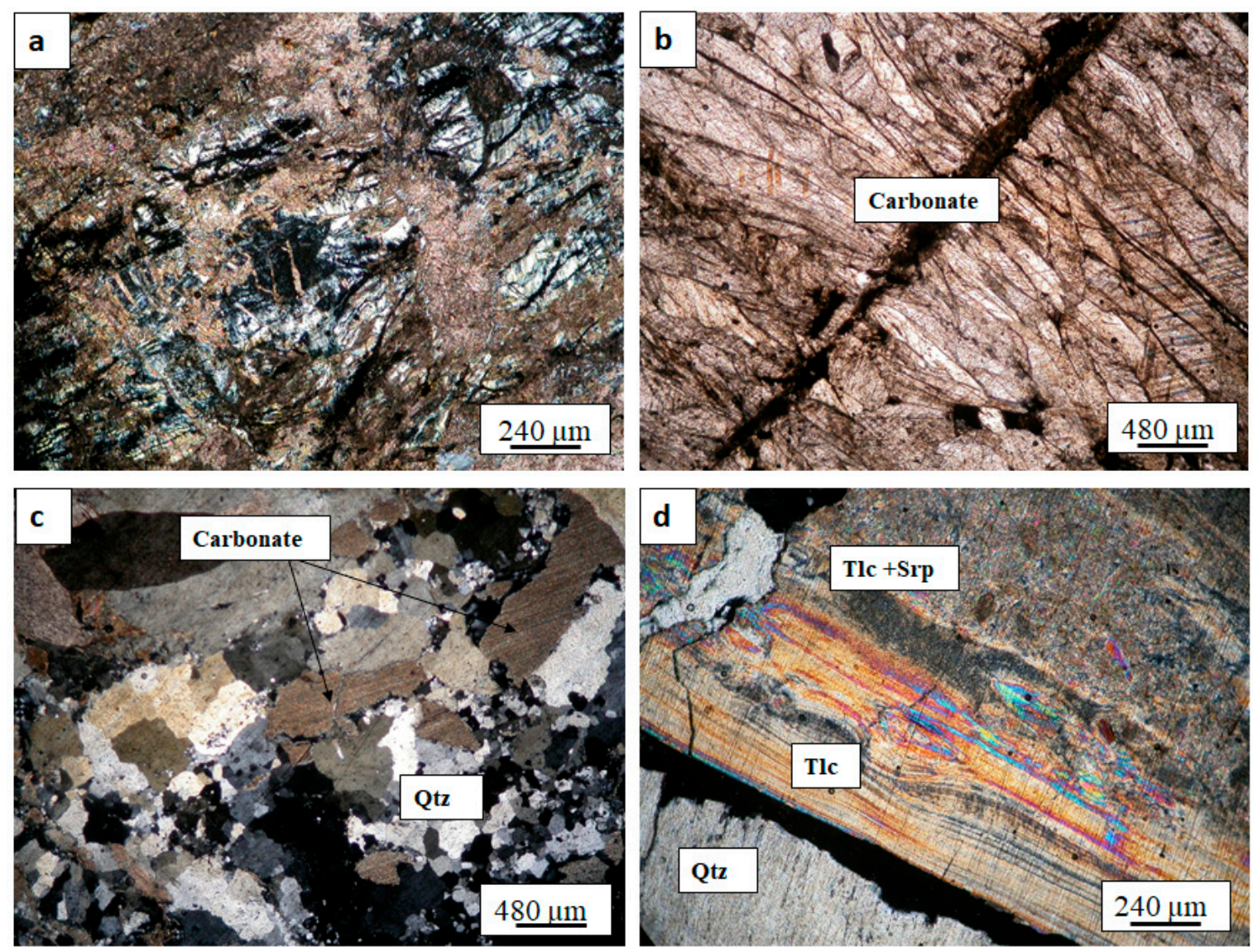

Figure 4. Photomicrographs of SpPP samples: (a) irregular patchy texture, crossed polarized light, $4 \times$; (b) carbonate veins with elongated rhombohedral crystals, plane polarized light, $4 \times$; (c) dynamic recrystallization of quartz, crossed polarized light, $4 \times$; (d) fibers of talc associated with serpentine and quartz, crossed polarized light, $4 \times$. Qtz = quartz; Srp = serpentine; Tlc = talc. Abbreviation after [72]. 


\subsection{Mineral Chemistry}

To better characterize silicate and carbonate minerals, an EMPA analysis was performed on selected samples of serpentinites and associated veins, from Fosso Arcangelo (SpFA5 and SpFA39) and Pietrapica (SpPP34A) sites. Amphiboles were analyzed in the host rock and associated veins, pyroxene and garnet were analyzed in the host rock, carbonate minerals were analyzed in the veins only (Tables 2-5).

The chemical composition of amphiboles is Ca-amphiboles [66], including tremolite and magnesio-ferro-hornblende (Table 2). EMP analyses of edenite crystals are reported by [73].

Garnet is a Ti-rich hydro-andradite $\left(\mathrm{TiO}_{2}=0.75-3.59 \mathrm{wt} \%\right)$ with high $\mathrm{Fe}_{\text {tot }}, \mathrm{Ca}$, and $\mathrm{Mg}$ contents $\left(\mathrm{FeO}_{\text {tot }}=17.49-23.41 \mathrm{wt} \% ; \mathrm{CaO}=27.57-32.88 \mathrm{wt} \% ; \mathrm{MgO}=1.41-6.50 \mathrm{wt} \%\right)$ and low $\mathrm{Al}$ content $\left(\mathrm{Al}_{2} \mathrm{O}_{3}\right.$ $=0.95-1.97 \mathrm{wt} \%)$ (Table 3, Figure 5).

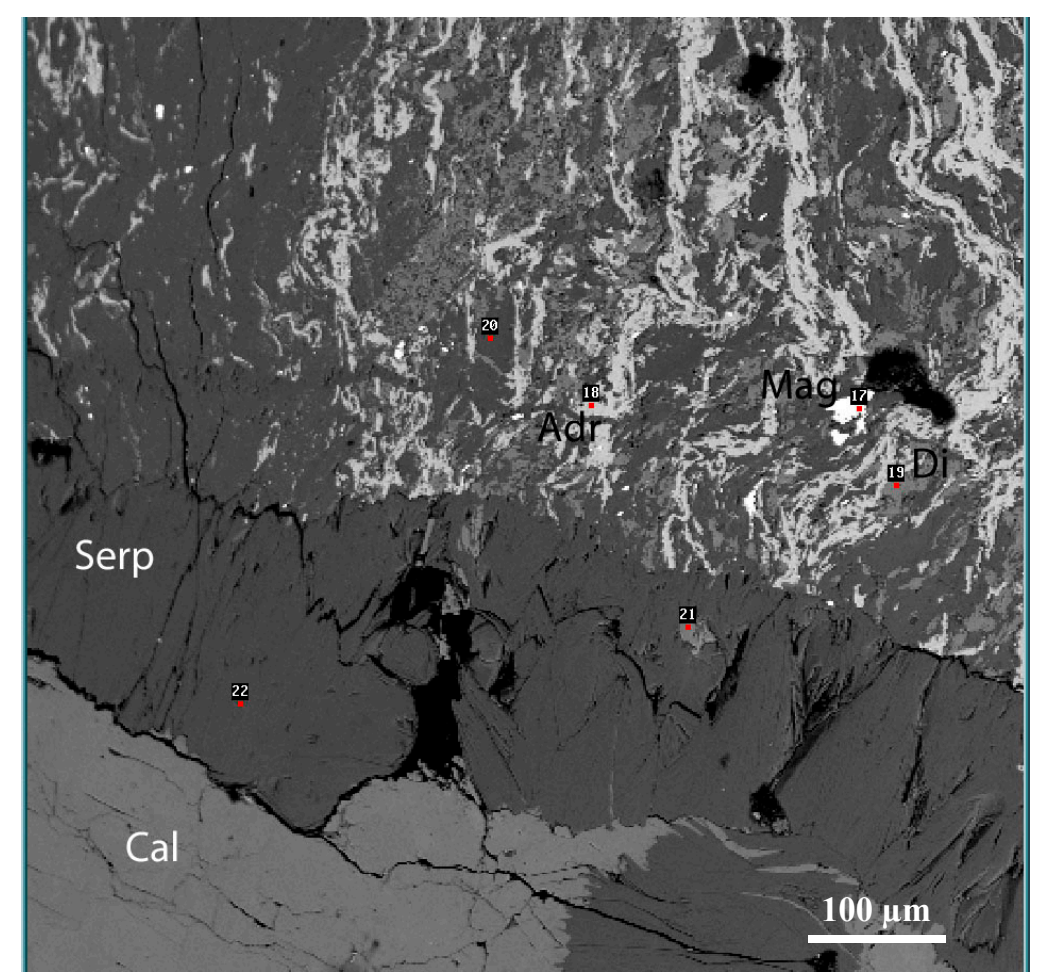

Figure 5. Secondary-electron image of serpentinite SpFA showing the presence of andradite (Adr), diopside (Di), and small crystals of magnetite (Mag) analyzed by EMPA. Adr = andradite; Cal = calcite; $\mathrm{Di}=$ diopside; $\mathrm{Mag}=$ magnesite; Serp = serpentine.

Clinopyroxene is characterized by a homogeneous composition rich in the diopside end-member $[78,79]$, with high Fe content $(\mathrm{FeO}=0.88-2.85 \mathrm{wt} \%)$ and usually low $\mathrm{Al}\left(\mathrm{Al}_{2} \mathrm{O}_{3}=\right.$ 0.03-1.53 wt \%) and $\mathrm{Cr}$ amounts $\left(\mathrm{Cr}_{2} \mathrm{O}_{3}=0.01-0.16\right.$ wt \%) (Table 4, Figure 5).

Carbonates consist of calcite $(\mathrm{CaO}=52.05-62.38 \mathrm{wt} \%), \mathrm{Mg}$-calcite $(\mathrm{CaO}=54.81-55.55 \mathrm{wt} \% ; \mathrm{MgO}$ $=1.26 \mathrm{wt} \%)$, and dolomite $\left(\mathrm{CaO}=29.60-29.79 \mathrm{wt} \% ; \mathrm{MgO}=19.06-19.23 \mathrm{wt} \%\right.$; and $\mathrm{FeO}_{\text {tot }}=2.17-3.68$ wt \%) (Table 5). 
Table 2. Chemical analyses of amphiboles in serpentinites and associated veins (v subscript) of Fosso Arcangelo (SpFA39, SpFA5).

\begin{tabular}{|c|c|c|c|c|c|c|c|c|c|c|}
\hline Sample Code & SpFA39 $_{v}$ & SpFA39 $_{v}$ & $\mathrm{SpFA5}_{\mathrm{v}}$ & $\mathrm{SpFA5}_{\mathrm{v}}$ & SpFA5 $_{v}$ & SpFA5 $_{v}$ & SpFA39 & SpFA39 & SpFA5v & SpFA5v \\
\hline No. Analysis & 73 & 76 & 77 & 78 & 79 & 91 & 98 & 102 & 109 & 130 \\
\hline \multicolumn{11}{|c|}{ Oxides (wt \%) } \\
\hline $\mathrm{SiO}_{2}$ & 54.588 & 57.392 & 55.674 & 52.04 & 53.735 & 57.547 & 51.657 & 55.263 & 55.015 & 57.337 \\
\hline $\mathrm{P}_{2} \mathrm{O}_{5}$ & 0.031 & n.d. & n.d. & 0.01 & 0.016 & 0.028 & n.d. & 0.005 & 0.024 & 0.057 \\
\hline $\mathrm{TiO}_{2}$ & 0.158 & 0.011 & 0.059 & 0.433 & 0.268 & 0.075 & 0.482 & 0.065 & 0.059 & n.d. \\
\hline $\mathrm{Al}_{2} \mathrm{O}_{3}$ & 2.798 & 0.479 & 1.559 & 5.282 & 3.543 & 1.369 & 5.484 & 1.871 & 2.509 & n..d. \\
\hline $\mathrm{Cr}_{2} \mathrm{O}_{3}$ & 0.224 & 0.007 & 0.092 & 0.425 & 0.486 & 0.009 & 0.502 & 0.006 & 0.225 & n.d. \\
\hline $\mathrm{MnO}$ & 0.138 & 0.089 & 0.045 & 0.026 & 0.119 & 0.082 & 0.03 & 0.17 & 0.082 & 0.022 \\
\hline $\mathrm{FeO}$ & 3.925 & 3.147 & 2.475 & 3.231 & 3.064 & 2.663 & 2.871 & 7.065 & 2.401 & 2.012 \\
\hline $\mathrm{NiO}$ & 0.108 & 0.09 & 0.05 & 0.082 & 0.076 & 0.045 & 0.139 & 0.05 & n.d. & n.d. \\
\hline $\mathrm{MgO}$ & 23.1 & 23.415 & 24.408 & 22.602 & 23.236 & 23.524 & 21.81 & 20.999 & 23.623 & 23.445 \\
\hline $\mathrm{CaO}$ & 11.427 & 13.574 & 12.845 & 11.959 & 12.063 & 12.273 & 12.421 & 9.68 & 12.523 & 13.653 \\
\hline $\mathrm{Na}_{2} \mathrm{O}$ & 1.192 & 0.09 & 0.459 & 1.187 & 1.144 & 0.358 & 1.296 & 1.995 & 0.813 & 0.07 \\
\hline $\mathrm{K}_{2} \mathrm{O}$ & 0.002 & n.d. & 0.014 & 0.008 & 0.014 & 0.014 & 0.028 & 0.015 & 0.016 & 0.022 \\
\hline $\mathrm{F}$ & n.d. & 0.093 & n.d. & 0.039 & 0.023 & n.d. & n.d. & n.d. & 0.037 & 0.032 \\
\hline $\mathrm{Cl}$ & n.d. & 0.018 & 0.019 & 0.004 & 0.02 & 0.011 & 0.027 & 0.003 & 0.006 & 0.01 \\
\hline Sum & 97.691 & 98.362 & 97.695 & 97.311 & 97.792 & 97.996 & 96.741 & 97.186 & 97.316 & 96.645 \\
\hline $\mathrm{Fe}^{3+/} \Sigma \mathrm{Fe}$ used & 1.000 & 0.970 & 1.000 & 1.000 & 1.000 & 0.798 & 1.000 & 1.000 & 1.000 & 0.827 \\
\hline $\mathrm{Mn}^{3+} / \Sigma \mathrm{Mn}$ used & 1.000 & 0.000 & 1.000 & 1.000 & 1.000 & 0.000 & 1.000 & 1.000 & 1.000 & 0.000 \\
\hline \multicolumn{11}{|c|}{ Final wt $\%$} \\
\hline $\mathrm{MnO}$ & 0.00 & 0.09 & 0.00 & 0.00 & 0.00 & 0.08 & 0.00 & 0.00 & 0.00 & 0.02 \\
\hline $\mathrm{Mn}_{2} \mathrm{O}_{3}$ & 0.15 & 0.00 & 0.05 & 0.03 & 0.13 & 0.00 & 0.03 & 0.19 & 0.09 & 0.00 \\
\hline $\mathrm{FeO}$ & 0.00 & 0.09 & 0.00 & 0.00 & 0.00 & 0.54 & 0.00 & 0.00 & 0.00 & 0.35 \\
\hline $\mathrm{Fe}_{2} \mathrm{O}_{3}$ & 4.36 & 3.39 & 2.75 & 3.59 & 3.41 & 2.36 & 3.19 & 7.85 & 2.67 & 1.85 \\
\hline $\mathrm{H}_{2} \mathrm{O}^{+}$ & 2.14 & 2.14 & 2.17 & 2.05 & 2.10 & 2.18 & 2.05 & 2.15 & 2.15 & 2.18 \\
\hline Sum & 100.28 & 100.88 & 100.15 & 99.74 & 100.26 & 100.42 & 99.12 & 100.14 & 99.76 & \\
\hline Group & $\mathrm{OH}, \mathrm{F}, \mathrm{Cl}$ & $\mathrm{OH}, \mathrm{F}, \mathrm{Cl}$ & $\mathrm{OH}, \mathrm{F}, \mathrm{Cl}$ & $\mathrm{OH}, \mathrm{F}, \mathrm{Cl}$ & $\mathrm{OH}, \mathrm{F}, \mathrm{Cl}$ & $\mathrm{OH}, \mathrm{F}, \mathrm{Cl}$ & $\mathrm{OH}, \mathrm{F}, \mathrm{Cl}$ & $\mathrm{OH}, \mathrm{F}, \mathrm{Cl}$ & $\mathrm{OH}, \mathrm{F}, \mathrm{Cl}$ & $\mathrm{OH}, \mathrm{F}, \mathrm{Cl}$ \\
\hline Subgroup of $(\mathrm{OH}, \mathrm{F}, \mathrm{Cl})$ & $\mathrm{Ca}$ & $\mathrm{Ca}$ & $\mathrm{Ca}$ & $\mathrm{Ca}$ & $\mathrm{Ca}$ & $\mathrm{Ca}$ & $\mathrm{Ca}$ & $\mathrm{Ca}$ & $\mathrm{Ca}$ & $\mathrm{Ca}$ \\
\hline Species & $\operatorname{Tr}$ & $\mathrm{Tr}$ & $\operatorname{Tr}$ & $\mathrm{Tr}$ & $\mathrm{Tr}$ & $\mathrm{Tr}$ & $\mathrm{Mg}-\mathrm{Fe} 2-\mathrm{H}$ & $1 \mathrm{Mg}-\mathrm{Fe} 2-\mathrm{Hbl}$ & $\operatorname{Tr}$ & $\mathrm{Tr}$ \\
\hline
\end{tabular}


Table 2. Cont.

\begin{tabular}{|c|c|c|c|c|c|c|c|c|c|c|}
\hline Sample Code & SpFA39 $_{v}$ & SpFA39 $_{v}$ & $\mathrm{SpFA}_{\mathrm{v}}$ & $\mathrm{SpFA}_{\mathrm{v}}$ & SpFA5 & $\mathrm{SpFA5}_{\mathrm{v}}$ & SpFA39 & SpFA39 & SpFA5v & SpFA5v \\
\hline No. Analysis & 73 & 76 & 77 & 78 & 79 & 91 & 98 & 102 & 109 & 130 \\
\hline \multicolumn{11}{|c|}{ T (ideally 8 apfu) } \\
\hline $\mathrm{Si}$ & 7.493 & 7.800 & 7.619 & 7.207 & 7.389 & 7.813 & 7.209 & 7.628 & 7.561 & 7.907 \\
\hline $\mathrm{P}$ & 0.002 & 0.000 & 0.000 & 0.001 & 0.001 & 0.002 & 0.000 & 0.000 & 0.001 & 0.003 \\
\hline $\mathrm{Al}$ & 0.453 & 0.077 & 0.251 & 0.792 & 0.574 & 0.185 & 0.791 & 0.304 & 0.406 & 0.000 \\
\hline $\mathrm{Ti}$ & 0.016 & 0.001 & 0.006 & 0.000 & 0.028 & 0.000 & 0.000 & 0.007 & 0.006 & 0.000 \\
\hline $\mathrm{Fe}^{3+}$ & 0.036 & 0.123 & 0.123 & 0.000 & 0.008 & 0.000 & 0.000 & 0.061 & 0.025 & 0.090 \\
\hline T subtotal & 8.000 & 8.001 & 7.999 & 8.000 & 8.000 & 8.000 & 8.000 & 8.000 & 7.999 & 8.000 \\
\hline \multicolumn{11}{|c|}{ C (ideally 5 apfu) } \\
\hline $\mathrm{Ti}$ & 0.000 & 0.000 & 0.000 & 0.045 & 0.000 & 0.008 & 0.051 & 0.000 & 0.000 & 0.000 \\
\hline $\mathrm{Al}$ & 0.000 & 0.000 & 0.000 & 0.070 & 0.000 & 0.034 & 0.111 & 0.000 & 0.000 & 0.000 \\
\hline $\mathrm{Cr}$ & 0.024 & 0.001 & 0.010 & 0.047 & 0.053 & 0.001 & 0.055 & 0.001 & 0.024 & 0.000 \\
\hline $\mathrm{Mn}^{3+}$ & 0.016 & 0.000 & 0.005 & 0.003 & 0.014 & 0.000 & 0.004 & 0.020 & 0.010 & 0.000 \\
\hline $\mathrm{Fe}^{3+}$ & 0.414 & 0.225 & 0.160 & 0.374 & 0.344 & 0.241 & 0.335 & 0.755 & 0.251 & 0.102 \\
\hline $\mathrm{Ni}$ & 0.012 & 0.010 & 0.006 & 0.009 & 0.008 & 0.005 & 0.016 & 0.006 & 0.000 & 0.000 \\
\hline $\mathrm{Mn}^{2+}$ & 0.000 & 0.010 & 0.000 & 0.000 & 0.000 & 0.000 & 0.000 & 0.000 & 0.000 & 0.003 \\
\hline $\mathrm{Fe}^{2+}$ & 0.000 & 0.011 & 0.000 & 0.000 & 0.000 & 0.000 & 0.000 & 0.000 & 0.000 & 0.000 \\
\hline $\mathrm{Mg}$ & 4.534 & 4.744 & 4.819 & 4.452 & 4.581 & 4.711 & 4.429 & 4.219 & 4.715 & 4.820 \\
\hline C subtotal & 5.000 & 5.001 & 5.000 & 5.000 & 5.000 & 5.000 & 5.001 & 5.001 & 5.000 & 4.965 \\
\hline \multicolumn{11}{|c|}{ B (ideally 2 apfu) } \\
\hline $\mathrm{Mn}^{2+}$ & 0.000 & 0.000 & 0.000 & 0.000 & 0.000 & 0.009 & 0.000 & 0.000 & 0.000 & 0.000 \\
\hline $\mathrm{Fe}^{2+}$ & 0.000 & 0.000 & 0.000 & 0.000 & 0.000 & 0.061 & 0.000 & 0.000 & 0.000 & 0.000 \\
\hline $\mathrm{Mg}$ & 0.193 & 0.000 & 0.160 & 0.214 & 0.182 & 0.050 & 0.108 & 0.102 & 0.125 & 0.000 \\
\hline $\mathrm{Ca}$ & 1.681 & 1.977 & 1.840 & 1.775 & 1.777 & 1.785 & 1.857 & 1.432 & 1.844 & 2.000 \\
\hline $\mathrm{Na}$ & 0.126 & 0.023 & 0.000 & 0.011 & 0.041 & 0.094 & 0.035 & 0.467 & 0.031 & 0.000 \\
\hline B subtotal & 2.000 & 2.000 & 2.000 & 2.000 & 2.000 & 1.999 & 2.000 & 2.001 & 2.000 & 2.000 \\
\hline
\end{tabular}


Table 2. Cont.

\begin{tabular}{ccccccccccc}
\hline Sample Code & SpFA39 $_{\mathbf{v}}$ & SpFA39 $_{\mathbf{v}}$ & SpFA5 $_{\mathbf{v}}$ & SpFA5 $_{\mathbf{v}}$ & SpFA5 $_{\mathbf{v}}$ & SpFA5 $_{\mathbf{v}}$ & SpFA39 $^{\text {SpFA39 }}$ & SpFA5v $^{\text {SpFA5v }}$ \\
\hline No. Analysis & 73 & 76 & 77 & 78 & 79 & 91 & 98 & 102 & 109 \\
\hline Ca & 0.000 & 0.000 & 0.044 & 0.000 & 0.000 & 0.000 & 0.000 & 0.000 & 0.000 & 0.017 \\
Na & 0.191 & 0.000 & 0.122 & 0.308 & 0.264 & 0.000 & 0.316 & 0.067 & 0.186 & 0.019 \\
K & 0.000 & 0.000 & 0.002 & 0.001 & 0.002 & 0.002 & 0.005 & 0.003 & 0.003 & 0.004 \\
A subtotal & 0.191 & 0.000 & 0.168 & 0.309 & 0.266 & 0.002 & 0.321 & 0.070 & 0.189 & 0.040 \\
O (non-W) & 22.000 & 22.000 & 22.000 & 22.000 & 22.000 & 22.000 & 22.000 & 22.000 & 22.000 & 22.000 \\
OH & 1.967 & 1.954 & 1.983 & 1.892 & 1.930 & 1.982 & 1.892 & 1.986 & 1.970 & 1.984 \\
F & 0.000 & 0.040 & 0.000 & 0.017 & 0.010 & 0.000 & 0.000 & 0.000 & 0.016 & 0.014 \\
Cl & 0.000 & 0.004 & 0.004 & 0.001 & 0.005 & 0.003 & 0.006 & 0.001 & 0.001 & 0.002 \\
O & 0.033 & 0.002 & 0.012 & 0.090 & 0.056 & 0.015 & 0.101 & 0.014 & 0.012 & n.d. \\
W subtotal & 2.000 & 2.000 & 1.999 & 2.000 & 2.001 & 2.000 & 1.999 & 2.001 & 1.999 & 2.000 \\
Sum T,C,B,A & 15.191 & 15.002 & 15.167 & 15.309 & 15.266 & 15.001 & 15.322 & 15.072 & 15.188 & 15.005 \\
\hline
\end{tabular}

Note: $\mathrm{Hbl}=$ hornblende; $\mathrm{Tr}=$ tremolite 
Table 3. Chemical analyses of Ti-rich hydro-andradites in a sample (SpFA39) of Fosso Arcangelo serpentinite.

\begin{tabular}{|c|c|c|c|c|c|}
\hline Sample code & SpFA39 & SpFA39 & SpFA39 & SpFA39 & SpFA39 \\
\hline N. Analysis & 8 & 18 & 26 & 29 & 48 \\
\hline \multicolumn{6}{|l|}{ Oxides (wt \%) } \\
\hline $\mathrm{SiO}_{2}$ & 34.90 & 33.68 & 34.28 & 34.17 & 34.67 \\
\hline $\mathrm{TiO}_{2}$ & 3.55 & 2.75 & 3.1 & 3.56 & 3.41 \\
\hline $\mathrm{Al}_{2} \mathrm{O}_{3}$ & 3.90 & 2.95 & 2.94 & 3.65 & 3.97 \\
\hline $\mathrm{MnO}$ & 0.06 & 0.12 & 0.07 & 0.09 & b.l.d. \\
\hline $\mathrm{Fe}_{2} \mathrm{O}_{3}$ & 20.27 & 23.41 & 21.49 & 21.32 & 20.88 \\
\hline $\mathrm{MgO}$ & 0.94 & 0.41 & 0.50 & 0.85 & 0.64 \\
\hline $\mathrm{CaO}$ & 33.47 & 33.49 & 34.57 & 33.87 & 33.85 \\
\hline $\mathrm{Na}_{2} \mathrm{O}$ & 0.01 & 0.02 & 0.01 & 0.01 & 0.03 \\
\hline $\mathrm{K}_{2} \mathrm{O}$ & n.d. & n.d. & b.l.d. & b.l.d. & b.l.d. \\
\hline $\mathrm{H}_{2} \mathrm{O}^{*}$ & 1.45 & 1.36 & 1.34 & 1.37 & 1.40 \\
\hline Sum & 98.55 & 98.19 & 98.30 & 98.89 & 98.85 \\
\hline \multicolumn{6}{|l|}{ Structural formula } \\
\hline $\mathrm{Si}$ & 5.672 & 5.510 & 5.610 & 5,582 & 5.650 \\
\hline $\mathrm{Ti}$ & 0.470 & 0.412 & 0.445 & 0.472 & 0.467 \\
\hline $\mathrm{Al}$ & 0.740 & 0.640 & 0.634 & 0.705 & 0.745 \\
\hline $\mathrm{Fe}$ & 2.770 & 2.988 & 2.899 & 2.831 & 2.779 \\
\hline Mn & 0.010 & 0.020 & 0.011 & 0.013 & 0.000 \\
\hline $\mathrm{Mg}$ & 0.172 & 0.106 & 0.110 & 0.156 & 0.131 \\
\hline $\mathrm{Ca}$ & 5.878 & 5.898 & 6.002 & 5.950 & 5.932 \\
\hline $\mathrm{H} / 4$ & 0.349 & 0.329 & 0.319 & 0.334 & 0.343 \\
\hline Sum & 16.061 & 15.903 & 16.030 & 16.043 & 16.047 \\
\hline Species & Hy-adr & Hy-adr & Hy-adr & Hy-adr & Hy-adr \\
\hline
\end{tabular}

Note: n.d. = not-detected; b.l.d. = below limit detection. $\mathrm{H}_{2} \mathrm{O}^{*}$ determined from stoichiometry. $\mathrm{Hy}$-adr = hydro-andradite. 
Table 4. Analyses of clinopyroxene in serpentinite of Fosso Arcangelo (SpFA39, SpFA5).

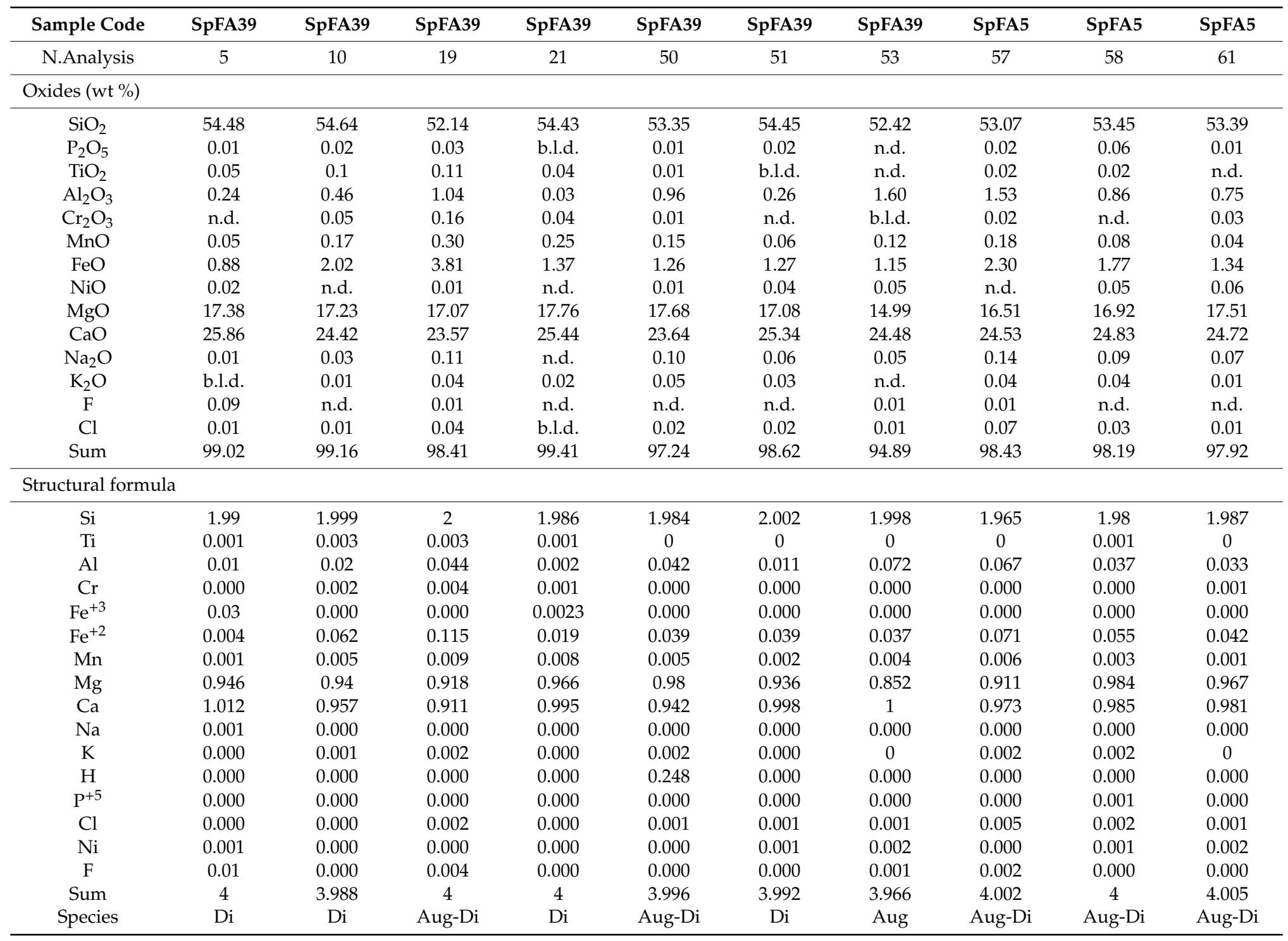


Table 4. Cont.

\begin{tabular}{|c|c|c|c|c|c|c|c|c|c|}
\hline Sample Code & SpFA39 & SpFA39 & SpFA39 & SpFA39 & \multicolumn{2}{|c|}{ SpFA39 } & SpFA39 & SpFA39 & SpFA39 \\
\hline N.Analysis & 62 & 63 & 69 & 70 & \multicolumn{2}{|c|}{78} & 97 & 98 & 104 \\
\hline \multicolumn{10}{|l|}{ Oxides (wt \%) } \\
\hline $\mathrm{P}_{2} \mathrm{O}_{5}$ & & 0.01 & 0.01 & 0.01 & b.l.d. & 0.05 & 0.05 & n.d. & 0.08 \\
\hline $\mathrm{TiO}_{2}$ & & b.l.d. & 0.01 & 0.01 & 0.02 & 0.02 & n.d. & 0.05 & 0.03 \\
\hline $\mathrm{Al}_{2} \mathrm{O}_{3}$ & & 1.75 & 0.47 & 0.78 & 0.38 & 0.39 & 0.03 & 0.56 & 0.14 \\
\hline $\mathrm{Cr}_{2} \mathrm{O}_{3}$ & & n.d. & 0.01 & 0.01 & b.l.d. & 0.01 & n.d. & n.d. & 0.01 \\
\hline $\mathrm{NiO}$ & & 0.02 & n.d. & 0.05 & 0.03 & 0.01 & 0.02 & n.d. & n.d. \\
\hline $\mathrm{MgO}$ & & 16.88 & 16.72 & 16.70 & 17.10 & 16.99 & 17.99 & 17.06 & 16.67 \\
\hline $\mathrm{CaO}$ & & 24.05 & 25.23 & 24.95 & 25.51 & 25.40 & 25.69 & 24.32 & 25.69 \\
\hline $\mathrm{Na}_{2} \mathrm{O}$ & & 0.11 & 0.08 & 0.06 & 0.10 & 0.06 & n.d. & 0.05 & n.d. \\
\hline $\mathrm{K}_{2} \mathrm{O}$ & & 0.04 & n.d. & 0.03 & n.d. & 0.01 & 0.01 & 0.01 & 0.01 \\
\hline $\mathrm{F}$ & & 0.02 & 0.02 & n.d. & n.d. & n.d. & 0.02 & 0.03 & 0.02 \\
\hline $\mathrm{Si}$ & & 1.918 & 1.994 & 1.976 & 1.993 & 1.976 & 1.989 & 1.983 & 1.989 \\
\hline $\mathrm{Ti}$ & & 0.000 & 0.000 & 0.000 & 0.001 & 0.001 & 0.000 & 0.001 & 0.001 \\
\hline $\mathrm{Al}$ & & 0.08 & 0.021 & 0.035 & 0.017 & 0.017 & 0.001 & 0.025 & 0.006 \\
\hline $\mathrm{Fe}^{+2}$ & & 0.054 & 0.057 & 0.061 & 0.044 & 0.087 & 0.032 & 0.066 & 0.06 \\
\hline $\mathrm{Mn}$ & & 0.003 & 0.004 & 0.004 & 0.004 & 0.004 & 0.003 & 0.01 & 0.009 \\
\hline $\mathrm{Mg}$ & & 0.978 & 0.918 & 0.929 & 0.936 & 0.929 & 0.977 & 0.946 & 0.918 \\
\hline $\mathrm{Ca}$ & & 1.001 & 0.996 & 0.998 & 1.003 & 0.998 & 1.003 & 0.969 & 1.017 \\
\hline K & & 0.002 & 0.000 & 0.001 & 0.000 & 0.000 & 0.001 & 0.000 & 0.001 \\
\hline $\mathrm{P}^{+5}$ & & 0.000 & 0.000 & 0.000 & 0.000 & 0.001 & 0.001 & 0.000 & 0.001 \\
\hline $\mathrm{Cl}$ & & 0.002 & 0.001 & 0.000 & 0.001 & 0.000 & 0.000 & 0.001 & 0.000 \\
\hline $\mathrm{Ni}$ & & 0.001 & 0.000 & 0.001 & 0.001 & 0.000 & 0.001 & 0.000 & 0.000 \\
\hline F & & 0.003 & 0.003 & 0.000 & 0.000 & 0.000 & 0.002 & 0.003 & 0.002 \\
\hline Sum & & 4.042 & 3.995 & 4.006 & 3.998 & 4.014 & 4.01 & 4.004 & 4.005 \\
\hline
\end{tabular}


Table 5. Analyses of carbonate minerals in veins of Fosso Arcangelo (SpFA39v, SpFA5v) and Pietrapica (SpPP34Av) sites.

\begin{tabular}{|c|c|c|c|c|c|c|c|c|c|c|c|c|c|c|c|}
\hline Sample Code & SpFA394v & SpFA39v & SpFA39v & SpFA39v & SpFA39v & SpFA39v & SpFA39v & SpFA39v & SpFA39v & SpFA39v & SpFA39v & SpFA39v & SpFA39v & SpFA39v & SpFA39v \\
\hline N.Analysis & 1 & 2 & 3 & 4 & 11 & 12 & 14 & 23 & 24 & 31 & 32 & 39 & 40 & 41 & 54 \\
\hline \multicolumn{16}{|l|}{ Oxides (wt \%) } \\
\hline $\mathrm{P}_{2} \mathrm{O}_{5}$ & n.d. & n.d. & n.d. & n.d. & 0.035 & 0.055 & 0.014 & 0.047 & 0.027 & 0.071 & 0.078 & 0.058 & 0.049 & 0.035 & 0.035 \\
\hline $\mathrm{TiO}_{2}$ & n.d. & n.d. & n.d. & n.d. & n.d. & n.d. & n.d. & n.d. & n.d. & 0.058 & n.d. & n.d. & 0.053 & 0.02 & 0.008 \\
\hline $\mathrm{Al}_{2} \mathrm{O}_{3}$ & n.d. & n.d. & n.d. & n.d. & 0.03 & 0.016 & n.d. & n.d. & 0.014 & 0.01 & n.d. & n.d. & 0.002 & 0.04 & n.d. \\
\hline $\mathrm{Cr}_{2} \mathrm{O}_{3}$ & n.d. & n.d. & n.d. & n.d. & n.d. & 0.031 & 0.076 & n.d. & 0.045 & 0.003 & n.d. & 0.002 & n.d. & n.d. & 0.001 \\
\hline $\mathrm{MnO}$ & 0.114 & 0.055 & n.d. & n.d. & n.d. & n.d. & 0.018 & n.d. & n.d. & 0.015 & n.d. & n.d. & 0.033 & 0.024 & 0.004 \\
\hline $\mathrm{FeO}$ & 1.78 & n.d. & n.d. & 0.005 & 0.113 & n.d. & n.d. & n.d. & n.d. & n.d. & n.d. & 0.103 & 0.042 & n.d. & n.d. \\
\hline $\mathrm{NiO}$ & n.d. & n.d. & n.d. & n.d. & n.d. & 0.095 & n.d. & 0.017 & 0.017 & n.d. & n.d. & 0.012 & 0.041 & 0.005 & 0.032 \\
\hline $\mathrm{MgO}$ & 18.706 & n.d. & n.d. & n.d. & n.d. & n.d. & 0.004 & n.d. & n.d. & n.d. & n.d. & n.d. & n.d. & n.d. & 0.059 \\
\hline $\mathrm{CaO}$ & 25.196 & 56.118 & 57.277 & 55.394 & 55.813 & 56.98 & 57.27 & 54.876 & 56.913 & 56.188 & 62.382 & 55.598 & 55.6 & 54.752 & 54.193 \\
\hline $\mathrm{SrO}$ & n.d. & 0.203 & 0.193 & 0.218 & n.d. & n.d. & n.d. & n.d. & n.d. & n.d. & n.d. & n.d. & n.d. & n.d. & n.d. \\
\hline $\mathrm{Na}_{2} \mathrm{O}$ & n.d. & n.d. & n.d. & n.d. & 0.015 & n.d. & 0.03 & 0.025 & 0.006 & 0.018 & n.d. & n.d. & n.d. & 0.041 & 0.013 \\
\hline $\mathrm{K}_{2} \mathrm{O}$ & n.d. & n.d. & n.d. & n.d. & 0.005 & n.d. & 0.032 & 0.002 & n.d. & 0.017 & 0.009 & n.d. & 0.009 & 0.01 & n.d. \\
\hline $\mathrm{F}$ & n.d. & n.d. & n.d. & n.d. & n.d. & n.d. & n.d. & 0.03 & n.d. & n.d. & n.d. & 0.018 & 0.026 & 0.049 & 0.003 \\
\hline $\mathrm{Cl}$ & n.d. & n.d. & n.d. & n.d. & 0.003 & 0.003 & 0.015 & 0.002 & 0.005 & n.d. & 0.005 & 0.002 & 0.009 & n.d. & 0.013 \\
\hline Sum & 45.796 & 56.376 & 57.47 & 55.617 & 56.013 & 57.179 & 57.456 & 54.986 & 57.026 & 56.38 & 62.473 & 55.785 & 55.851 & 54.955 & 54.357 \\
\hline $\mathrm{CO}_{2}^{*}$ & 41,36 & 44,16 & 45,03 & 43,57 & 44.03 & 44.86 & n.d. & 43.17 & 44.71 & 44.15 & 48.98 & 43.75 & 43.82 & 43.17 & 42.66 \\
\hline \multicolumn{16}{|c|}{ Structural formula } \\
\hline $\mathrm{Sr}$ & 0.000 & 0.01 & 0.01 & 0.01 & 0.01 & 0.000 & 0.000 & 0.000 & 0.000 & 0.04 & 0.000 & 0.000 & 0.000 & 0.000 & 0.000 \\
\hline $\mathrm{Ti}$ & 0.000 & 0.000 & 0.000 & 0.000 & 0.000 & 0.000 & 0.000 & 0.000 & 0.000 & 0.001 & 0.000 & 0.000 & 0.001 & 0.001 & 0.000 \\
\hline $\mathrm{Al}$ & 0.000 & 0.000 & 0.000 & 0.000 & 0.001 & 0.001 & 0.000 & 0.000 & 0.000 & 0.000 & 0.000 & 0.000 & 0.000 & 0.002 & 0.000 \\
\hline $\mathrm{Cr}$ & 0.000 & 0.000 & 0.000 & 0.000 & 0.000 & 0.000 & 0.002 & 0.000 & 0.001 & 0.000 & 0.000 & 0.000 & 0.000 & 0.000 & 0.000 \\
\hline $\mathrm{Fe}^{+2}$ & 0.000 & 0.000 & 0.000 & 0.000 & 0.003 & 0.000 & 0.000 & 0.000 & 0.000 & 0.000 & 0.000 & 0.003 & 0.001 & 0.000 & 0.000 \\
\hline $\mathrm{Mn}$ & 0.003 & 0.02 & 0.000 & 0.000 & 0.000 & 0.000 & 0.000 & 0.000 & 0.000 & 0.000 & 0.000 & 0.000 & 0.001 & 0.001 & 0.000 \\
\hline $\mathrm{Mg}$ & 0.988 & 0.000 & 0.000 & 0.000 & 0.000 & 0.000 & 0.000 & 0.000 & 0.000 & 0.000 & 0.000 & 0.000 & 0.000 & 0.000 & 0.003 \\
\hline $\mathrm{Ca}$ & 0.956 & 1.993 & 1.995 & 1.994 & 1.984 & 1.994 & 1.995 & 1.999 & 1.997 & 1.983 & 1.998 & 1.994 & 1.991 & 1.99 & 1.994 \\
\hline K & 0.000 & 0.000 & 0.000 & 0.000 & 0.000 & 0.000 & 0.001 & 0.000 & 0.000 & 0.001 & 0.000 & 0.000 & 0.000 & 0.000 & 0.000 \\
\hline $\mathrm{P}^{+5}$ & 0.000 & 0.000 & 0.000 & 0.000 & 0.000 & 0.001 & 0.000 & 0.000 & 0.000 & 0.001 & 0.001 & 0.001 & 0.001 & 0.001 & 0.001 \\
\hline $\mathrm{Cl}$ & 0.000 & 0.000 & 0.000 & 0.000 & 0.000 & 0.000 & 0.001 & 0.000 & 0.000 & 0.000 & 0.000 & 0.000 & 0.001 & 0.000 & 0.001 \\
\hline $\mathrm{Ni}$ & 0.000 & 0.000 & 0.000 & 0.000 & 0.000 & 0.002 & 0.000 & 0.000 & 0.000 & 0.000 & 0.000 & 0.000 & 0.001 & 0.000 & 0.001 \\
\hline $\mathrm{F}$ & 0.000 & 0.000 & 0.000 & 0.000 & 0.000 & 0.000 & 0.000 & 0.003 & 0.000 & 0.000 & 0.000 & 0.002 & 0.003 & 0.005 & 0.000 \\
\hline $\mathrm{C}$ & 2 & 1.999 & 1.999 & 1.999 & 1.999 & 2 & 1.999 & 2 & 1.999 & 1.986 & 1.999 & 1.999 & 1.999 & 1.999 & 2 \\
\hline Sum & 4 & 4.001 & 4.001 & 4.002 & 4.001 & 3.998 & 4 & 4 & 3.999 & 4.012 & 3.999 & 3.999 & 3.999 & 3.999 & 3.999 \\
\hline Species & Dol & Cal & Cal & Cal & Cal & Cal & Cal & Cal & Cal & Cal & Cal & Cal & Cal & Cal & Cal \\
\hline
\end{tabular}


Table 5. Cont.

\begin{tabular}{|c|c|c|c|c|c|c|c|c|c|c|c|c|c|c|}
\hline Sample Code & SpFA39v & SpFA39v & SpFA39v & SpFA39v & SpFA39v & SpFA39v & SpFA39v & SpFA39v & SpFA39v & SpFA5v & SpFA5v & SpFA5v & SpFA5v & SpFA5v \\
\hline N. Analysis & 55 & 56 & 59 & 60 & 66 & 73 & 85 & 87 & 92 & 46 & 51 & 65 & 70 & 86 \\
\hline \multicolumn{15}{|l|}{ Oxides (wt \%) } \\
\hline $\mathrm{P}_{2} \mathrm{O}_{5}$ & 0.032 & 0.06 & 0.057 & 0.065 & 0.052 & 0.076 & 0.054 & 0.054 & 0.011 & 0.078 & 0.065 & 0.034 & 0.067 & 0.075 \\
\hline $\mathrm{TiO}_{2}$ & 0.048 & 0.017 & 0.014 & n.d. & 0.141 & 0.04 & n.d. & n.d. & 0.017 & 0.036 & 0.028 & 0.024 & n.d. & 0.045 \\
\hline $\mathrm{Al}_{2} \mathrm{O}_{3}$ & 0.012 & n.d. & n.d. & n.d. & n.d. & n.d. & 0.226 & n.d. & n.d. & 0.05 & n.d. & 0.592 & n.d. & 0.021 \\
\hline $\mathrm{Cr}_{2} \mathrm{O}_{3}$ & n.d. & 0.003 & 0.095 & 0.033 & 0.02 & n.d. & 0.019 & n.d. & n.d. & n.d. & n.d. & 0.012 & n.d. & 0.016 \\
\hline $\mathrm{MnO}$ & n.d. & 0.046 & n.d. & 0.013 & 0.04 & 0.023 & n.d. & 0.035 & n.d. & n.d. & 0.023 & n.d. & 0.026 & 0.004 \\
\hline $\mathrm{FeO}$ & 0.014 & n.d. & 0.013 & 0.017 & 0.006 & n.d. & 0.017 & 0.13 & 0.227 & 0.099 & 0.048 & 0.025 & 0.003 & n.d. \\
\hline $\mathrm{NiO}$ & n.d. & n.d. & 0.005 & 0.039 & 0.005 & n.d. & n.d. & n.d. & n.d. & n.d. & 0.052 & 0.06 & n.d. & 0.008 \\
\hline $\mathrm{MgO}$ & n.d. & n.d. & n.d. & n.d. & n.d. & n.d. & 0.144 & 0.109 & 0.018 & 1.265 & n.d. & n.d. & n.d. & n.d. \\
\hline $\mathrm{CaO}$ & 54.686 & 54.332 & 54.804 & 54.074 & 53.64 & 55.502 & 55.12 & 52.047 & 44.394 & 54.813 & 57.314 & 55.63 & 56.344 & 56.791 \\
\hline $\mathrm{Na}_{2} \mathrm{O}$ & 0.01 & 0.016 & 0.03 & 0.028 & n.d. & 0.012 & n.d. & 0.037 & 0.037 & 0.019 & 0.012 & 0.059 & 0.001 & 0.026 \\
\hline $\mathrm{K}_{2} \mathrm{O}$ & 0.015 & n.d. & n.d. & 0.013 & 0.015 & 0.017 & 0.001 & 0.004 & 0.009 & n.d. & 0.036 & n.d. & n.d. & 0.009 \\
\hline $\mathrm{F}$ & 0.016 & 0.025 & 0.028 & n.d. & 0.04 & n.d. & 0.015 & n.d. & 0.016 & n.d. & 0.022 & n.d. & 0.014 & n.d. \\
\hline $\mathrm{Cl}$ & 0.016 & 0.007 & 0.005 & n.d. & 0.048 & n.d. & 0.005 & n.d. & 0.373 & 0.012 & n.d. & 0.006 & n.d. & n.d. \\
\hline Sum & 54.838 & 54.493 & 55.038 & 54.282 & 53.979 & 55.67 & 55.642 & 52.416 & 45,102 & 57.241 & 57.591 & 56.441 & 56.449 & 56.995 \\
\hline $\mathrm{CO}_{2} *$ & 43.03 & 42.72 & 43.16 & 42.52 & 42.38 & 43.62 & n.d. & 41.10 & 45.011 & 45.18 & 45.16 & 44.04 & 44.28 & 44.68 \\
\hline \multicolumn{15}{|c|}{ Structural formula } \\
\hline $\mathrm{Ti}$ & 0.001 & 0.000 & 0.000 & 0.000 & 0.004 & 0.001 & 0.000 & 0.000 & 0.001 & 0.001 & 0.001 & 0.001 & 0.000 & 0.001 \\
\hline $\mathrm{Al}$ & 0.000 & 0.000 & 0.000 & 0.000 & 0.000 & 0.000 & 0.000 & 0.000 & 0.000 & 0.002 & 0.000 & 0.023 & 0.000 & 0.001 \\
\hline $\mathrm{Cr}$ & 0.000 & 0.000 & 0.003 & 0.001 & 0.001 & 0.000 & 0.000 & 0.000 & 0.000 & 0.000 & 0.000 & 0.000 & 0.000 & 0.000 \\
\hline $\mathrm{Fe}^{+2}$ & 0.000 & 0.000 & 0.000 & 0.000 & 0.000 & 0.000 & 0.000 & 0.004 & 0.008 & 0.003 & 0.001 & 0.001 & 0.000 & 0.000 \\
\hline $\mathrm{Mn}$ & 0.000 & 0.001 & 0.000 & 0.000 & 0.001 & 0.001 & 0.000 & 0.001 & 0.000 & 0.000 & 0.001 & 0.000 & 0.001 & 0.000 \\
\hline $\mathrm{Mg}$ & 0.000 & 0.000 & 0.000 & 0.000 & 0.000 & 0.000 & 0.000 & 0.006 & 0.001 & 0.061 & 0.000 & 0.000 & 0.000 & 0.000 \\
\hline $\mathrm{Ca}$ & 1.994 & 1.996 & 1.992 & 1.995 & 1.985 & 1.996 & 1.981 & 1.987 & 1.961 & 1.894 & 1.992 & 1.975 & 1.997 & 1.994 \\
\hline K & 0.000 & 0.001 & 0.000 & 0.001 & 0.001 & 0.001 & 0.000 & 0.000 & 0.000 & 0.000 & 0.001 & 0.000 & 0.000 & 0.000 \\
\hline $\mathrm{P}^{+5}$ & 0.000 & 0.001 & 0.001 & 0.001 & 0.001 & 0.001 & 0.001 & 0.001 & 0.000 & 0.001 & 0.001 & 0.000 & 0.001 & 0.001 \\
\hline $\mathrm{Cl}$ & 0.001 & 0.000 & 0.000 & 0.000 & 0.003 & 0.000 & 0.000 & 0.000 & 0.026 & 0.001 & 0.000 & 0.000 & 0.000 & 0.000 \\
\hline $\mathrm{Ni}$ & 0.000 & 0.000 & 0.000 & 0.001 & 0.000 & 0.000 & 0.000 & 0.000 & 0.000 & 0.000 & 0.001 & 0.002 & 0.000 & 0.000 \\
\hline $\mathrm{F}$ & 0.002 & 0.000 & 0.000 & 0.000 & 0.004 & 0.000 & 0.000 & 0.000 & 0.002 & 0.000 & 0.002 & 0.000 & 0.001 & 0.001 \\
\hline $\mathrm{C}$ & 1.999 & 1.999 & 1.999 & 1.999 & 1.998 & 1.999 & 1.996 & 2 & 2 & 1.989 & 1.999 & 1.992 & 1.999 & 1.999 \\
\hline Sum & 3.999 & 3.999 & 3.999 & 3.999 & 3.997 & 3.999 & 3.997 & 3.999 & 3.999 & 3.979 & 3.999 & 3.995 & 3.999 & 3.998 \\
\hline Species & Cal & Cal & $\mathrm{Cal}$ & $\mathrm{Cal}$ & Cal & Cal & $\mathrm{Cal}$ & $\mathrm{Cal}$ & $\mathrm{Cal}$ & $\mathrm{Mg}-\mathrm{Cal}$ & $\mathrm{Cal}$ & Cal & $\mathrm{Cal}$ & Cal \\
\hline
\end{tabular}


Table 5. Cont.

\begin{tabular}{|c|c|c|c|c|c|c|c|c|c|c|c|c|c|c|}
\hline Sample Code & SpFA5v & SpFA5v & SpFA5v & SpFA5v & SpFA5v & SpFA5v & SpFA5v & SpFA5v & SpFA5v & SpFA5v & SpPP34A & SpPP34A & SpPP34A & SpPP34Av \\
\hline N.Analysis & 87 & 96 & 115 & 121 & 122 & 125 & 129 & 131 & 132 & 133 & 15 & 16 & 37 & 41 \\
\hline \multicolumn{15}{|l|}{ Oxides (wt \%) } \\
\hline $\mathrm{P}_{2} \mathrm{O}_{5}$ & 0.068 & 0.016 & 0.022 & 0.053 & 0.041 & 0.017 & 0.055 & 0.027 & 0.044 & 0.047 & 0.034 & n.d. & 0.035 & 0.007 \\
\hline $\mathrm{TiO}_{2}$ & 0.035 & n.d. & 0.022 & n.d. & 0.04 & n.d. & n.d. & 0.013 & n.d. & n.d. & 0.001 & 0.036 & 0.025 & n.d. \\
\hline $\mathrm{Al}_{2} \mathrm{O}_{3}$ & 0.016 & 0.02 & n.d. & 0.02 & 0.001 & n.d. & 0.007 & n.d. & 0.014 & n.d. & 0.447 & n.d. & 0.253 & n.d. \\
\hline $\mathrm{Cr}_{2} \mathrm{O}_{3}$ & n.d. & n.d. & n.d. & 0.016 & n.d. & 0.049 & n.d. & n.d. & 0.043 & 0.122 & n.d. & n.d. & 0.028 & 0.009 \\
\hline $\mathrm{MnO}$ & 0.116 & n.d. & 0.045 & n.d. & 0.041 & n.d. & 0.072 & 0.023 & 0.004 & n.d. & 0.223 & 0.167 & 0.355 & 0.231 \\
\hline $\mathrm{FeO}$ & 0.106 & 0.079 & 0.018 & n.d. & 0.01 & 0.01 & 0.008 & n.d. & 0.033 & 0.03 & 2.303 & 3.685 & 2.166 & 2.405 \\
\hline $\mathrm{NiO}$ & n.d. & n.d. & 0.052 & 0.044 & 0.041 & 0.01 & 0.018 & n.d. & n.d. & 0.021 & 0.031 & n.d. & n.d. & 0.049 \\
\hline $\mathrm{MgO}$ & 1.264 & n.d. & n.d. & 0.13 & n.d. & n.d. & n.d. & n.d. & n.d. & n.d. & 19.178 & 19.232 & 19.124 & 19.062 \\
\hline $\mathrm{CaO}$ & 55.551 & 56.642 & 55.904 & 56.896 & 55.968 & 57.21 & 58.56 & 56.413 & 56.831 & 57.205 & 29.598 & 29.653 & 29.794 & 29.685 \\
\hline $\mathrm{Na}_{2} \mathrm{O}$ & 0.014 & n.d. & 0.024 & 0.034 & 0.012 & 0.019 & n.d. & n.d. & 0.005 & 0.026 & 0.037 & 0.054 & n.d. & n.d. \\
\hline $\mathrm{K}_{2} \mathrm{O}$ & 0.013 & 0.004 & n.d. & 0.006 & n.d. & 0.035 & 0.029 & n.d. & 0.029 & 0.013 & 0.014 & 0.008 & n.d. & n.d. \\
\hline $\mathrm{F}$ & 0.012 & n.d. & 0.032 & 0.039 & n.d. & 0.143 & n.d. & n.d. & 0.035 & 0.053 & n.d. & n.d. & 0.013 & 0.076 \\
\hline $\mathrm{Cl}$ & n.d. & 0.005 & 0.002 & 0.001 & n.d. & 0.018 & n.d. & n.d. & n.d. & 0.006 & 0.005 & 0.013 & n.d. & n.d. \\
\hline Sum & 57.19 & 56.765 & 56.108 & 57.223 & 56.154 & 57.447 & 58.749 & 56.476 & 57.023 & 57.5 & 51.87 & 52.845 & 51.788 & 51.492 \\
\hline $\mathrm{CO}_{2} *$ & 45.19 & 44.52 & 44.05 & 45.60 & 44.2 & 45.31 & 46.04 & 44.30 & 44.75 & 45.12 & 45.98 & 46.71 & 45.98 & 45.94 \\
\hline \multicolumn{15}{|c|}{ Structural formula } \\
\hline $\mathrm{Ti}$ & 0.001 & 0.000 & 0.001 & 0.000 & 0.001 & 0.000 & 0.000 & 0.000 & 0.000 & 0.000 & 0.000 & 0.001 & 0.001 & 0.000 \\
\hline $\mathrm{Al}$ & 0.001 & 0.001 & 0.000 & 0.001 & 0.000 & 0.000 & 0.000 & 0.000 & 0.001 & 0.000 & 0.017 & 0.000 & 0.009 & 0.000 \\
\hline $\mathrm{Cr}$ & 0.000 & 0.000 & 0.000 & 0.000 & 0.000 & 0.001 & 0.000 & 0.000 & 0.001 & 0.003 & 0.000 & 0.000 & 0.001 & 0.000 \\
\hline $\mathrm{Fe}^{+2}$ & 0.003 & 0.002 & 0.001 & 0.000 & 0.000 & 0.000 & 0.000 & 0.000 & 0.001 & 0.001 & 0.061 & 0.097 & 0.058 & 0.064 \\
\hline $\mathrm{Mn}$ & 0.003 & 0.000 & 0.001 & 0.000 & 0.001 & 0.000 & 0.002 & 0.001 & 0.000 & 0.000 & 0.006 & 0.004 & 0.01 & 0.006 \\
\hline $\mathrm{Mg}$ & 0.061 & 0.000 & 0.000 & 0.034 & 0.000 & 0.000 & 0.000 & 0.000 & 0.000 & 0.000 & 0.908 & 0.899 & 0.907 & 0.906 \\
\hline $\mathrm{Ca}$ & 1.928 & 1.997 & 1.992 & 1.958 & 1.995 & 1.981 & 1.996 & 1.998 & 1.993 & 1.989 & 1.008 & 0.997 & 1.015 & 1.014 \\
\hline K & 0.001 & 0.000 & 0.000 & 0.000 & 0.000 & 0.001 & 0.001 & 0.000 & 0.001 & 0.001 & 0.000 & 0.000 & 0.000 & 0.000 \\
\hline $\mathrm{P}^{+5}$ & 0.001 & 0.000 & 0.000 & 0.001 & 0.001 & 0.000 & 0.000 & 0.000 & 0.001 & 0.001 & 0.001 & 0.000 & 0.000 & 0.000 \\
\hline $\mathrm{Cl}$ & 0.000 & 0.000 & 0.000 & 0.000 & 0.000 & 0.001 & 0.000 & 0.000 & 0.000 & 0.000 & 0.000 & 0.001 & 0.000 & 0.000 \\
\hline $\mathrm{Ni}$ & 0.000 & 0.000 & 0.001 & 0.001 & 0.001 & 0.000 & 0.000 & 0.000 & 0.000 & 0.001 & 0.001 & 0.000 & 0.000 & 0.001 \\
\hline $\mathrm{F}$ & 0.001 & 0.000 & 0.003 & 0.004 & 0.000 & 0.015 & 0.000 & 0.000 & 0.004 & 0.005 & 0.000 & 0.000 & 0.001 & 0.008 \\
\hline C & 1.999 & 2 & 2 & 1.999 & 1.999 & 2 & 1.999 & 2 & 1.999 & 1.999 & 1.994 & 2 & 1.996 & 2 \\
\hline Sum & 3.999 & 4 & 3.999 & 3.999 & 3.999 & 4 & 4 & 3.999 & 4 & 3.999 & 3.857 & 3.999 & 3.998 & 4 \\
\hline Species & $\mathrm{Mg}-\mathrm{Cal}$ & Cal & $\mathrm{Cal}$ & $\mathrm{Cal}$ & Cal & Cal & Cal & $\mathrm{Cal}$ & Cal & Cal & Dol & Dol & Dol & Dol \\
\hline
\end{tabular}

Note: n.d. $=$ non-detected $; \mathrm{CO}_{2}{ }^{*}=$ calculated from stoichiometry. $\mathrm{Cal}=$ calcite; $\mathrm{Dol}=$ dolomite. 


\subsection{Mineralogy}

According to petrographic observations, the XRD analysis revealed that serpentinite samples from both studied sites are made up of serpentine polimorphs, mainly lizardite and, in minor amounts, chrysotile and antigorite, amphibole-like minerals, mainly actinolite and tremolite, clinochlore, magnetite, and calcite. Diopside and hydro-andradite are also present in the SpFA only.

As for veins, a different mineralogical composition was detected for the two analyzed sample groups. The vein infill of the SpFA samples consists of prevalent calcite and traces of aragonite and rhodochrosite. In these samples, traces of silicate minerals, such as serpentine, actinolite, and tremolite, were also detected. The infill of veins traversing the SpPP is dominated by dolomite and Mg-calcite, with quartz as the sole silicate phase.

$\mu$-Raman spectroscopy has been used as a complementary technique to the X-ray diffraction analysis to better identify the carbonate minerals (calcite, aragonite, and dolomite) of both serpentinites and crosscutting veins. The optical vibrations are internal vibrations of the $\mathrm{CO}_{3}$ group (three Raman bands lying between 1500 and $700 \mathrm{~cm}^{-1}$ ) and external or lattice vibrations involving translation and librations of the $\mathrm{CO}_{3}$ groups relative to the $\mathrm{Ca}$ or $\mathrm{Mg}$ atoms $\left(500-100 \mathrm{~cm}^{-1}\right)$ [80]. In our samples, calcite is characterized by a dominant Raman band at $1091 \mathrm{~cm}^{-1}$, minor bands at 713,280, and $155 \mathrm{~cm}^{-1}$ and a very weak band at $1439 \mathrm{~cm}^{-1}$ (Figure 6a). The Raman spectrum for aragonite signals are detected for a dominant Raman band at $1086 \mathrm{~cm}^{-1}$, two strong bands at 212 and $150 \mathrm{~cm}^{-1}$, and three weak bands at 703, 250, and $180 \mathrm{~cm}^{-1}$ (Figure 6b). In dolomite, the main peak in the Raman spectrum occurs at $1103 \mathrm{~cm}^{-1}$, whereas the weak peaks are at 730, 305, and $180 \mathrm{~cm}^{-1}$ (Figure 6c).

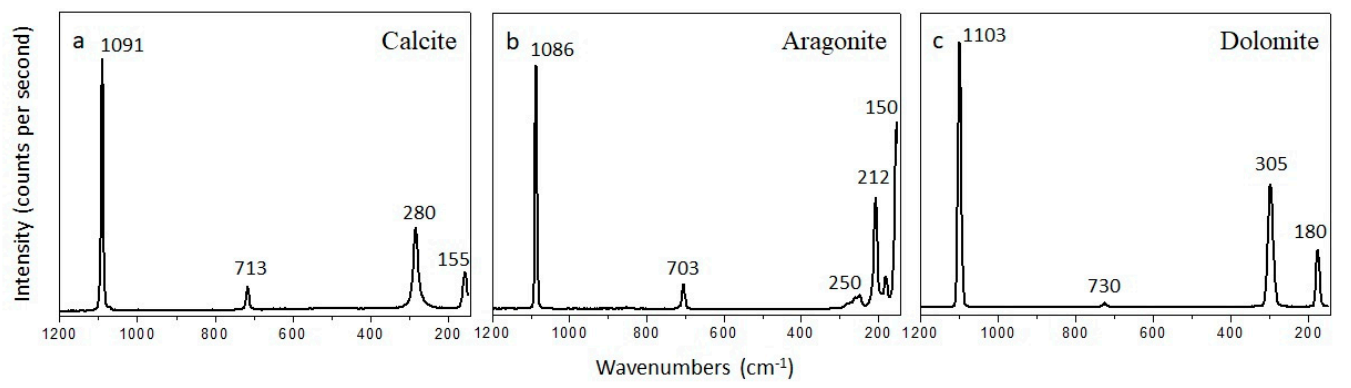

Figure 6. Raman spectra at low wavenumbers of carbonate minerals: (a) calcite; (b) aragonite; (c) dolomite in selected veins from SpFA and SpPP.

\subsection{Carbon and Oxygen Stable Isotope Analyses}

Results of isotope analyses of carbonate phases in the veins of SpFA and SpPP are presented in Tables 6 and 7.

The $\mathrm{C}$ and $\mathrm{O}$ isotope ratios of carbonates from the veins of the SpFA have two distinct ranges. The $\delta^{13} \mathrm{C}$ values range from $-0.81 \%$ o to $+2.16 \%$ o and from $-2.78 \%$ o to $-3.66 \%$ o vs. V-PDB, corresponding $\delta^{18} \mathrm{O}$ values are between $+15.02 \%$ ond $+16.38 \%$ o vs. V-SMOW, except for three samples (SpFA7av, SpFA30v, SpFA10v) having $\delta^{18} \mathrm{O}$ values slightly more positive (between $+18.36 \%$ o and $+21.53 \%$ o vs. $\mathrm{V}-\mathrm{SMOW})$. The $\delta^{13} \mathrm{C}$ values of carbonate veins of the SpPP are in the range from $-1.78 \%$ o to $-3.60 \%$ o vs. V-PDB, and the corresponding $\delta^{18} \mathrm{O}$ average value is $+21.3 \%$ o $(1 \sigma=0.18 ; n=6)$ vs. V-SMOW.

Based on the XRPD results, calcite and dolomite have been considered as dominant carbonate phases in the veins of the SpFA and SpPP, respectively. Accordingly, equilibrium temperatures for SpFA veins were computed from $\delta^{18} \mathrm{O}$ data and considering the calcite-water fractionation curves of $\mathrm{O}^{\prime} \mathrm{Neil}$ et al. [81], Friedman and $\mathrm{O}^{\prime} \mathrm{Neil}$ [82], and Kim and $\mathrm{O}^{\prime} \mathrm{Neil}$ [83]. Instead, for vein samples of SpPP, dolomite-water fractionation curves of Schmidt et al. [84] and Horita [85] have been considered. In both areas, the oxygen isotope composition of water $\left(\delta^{18} \mathrm{O}\right)$ has been assumed to be $0 \%$ similar to modern seawater, as used by Agrinier et al. [86]. Depending on the fractionation factors available from the literature, for the vein samples of SpFA we have obtained an equilibrium temperature ranging from 83 to $117^{\circ} \mathrm{C}\left(\Delta^{18} \mathrm{O}_{\text {calcite }}\right.$ from [81]), from 81 to $121^{\circ} \mathrm{C}\left(\Delta^{18} \mathrm{O}_{\text {calcite }}\right.$ from [82]), and from 76 to $107^{\circ} \mathrm{C}$ $\left(\Delta^{18} \mathrm{O}_{\text {calcite }}\right.$ from [83]). 
Table 6. $\mathrm{C}$ and $\mathrm{O}$ isotope data and calculated fluid isotopic composition for the calcite in serpentinites from the Fosso Arcangelo site.

\begin{tabular}{|c|c|c|c|c|c|c|c|c|c|c|c|}
\hline Sample No. & $\begin{array}{c}\text { Sample } \\
\text { Code }\end{array}$ & $\begin{array}{c}\text { Carbonate } \\
\text { Phase }\end{array}$ & $\begin{array}{c}\delta^{13} C_{\text {PDB }} \\
(\% \text { oo })\end{array}$ & $\begin{array}{c}\delta^{18} \text { OPDB }_{\text {PDB }} \\
(\% \text { o })\end{array}$ & $\begin{array}{c}\mathcal{\delta}^{18} \mathrm{O}_{\text {SMOW }} \\
(\%)^{\mathbf{a})}\end{array}$ & $\mathbf{T}^{\circ} \mathbf{C}^{\mathbf{b})}$ & $\mathrm{T}^{\circ} \mathrm{C}^{\mathrm{c})}$ & $T^{\circ} C^{d)}$ & $\begin{array}{c}\delta^{13} \mathrm{C}_{\mathrm{CO} 2} \text { fluid } \\
(\% \text { e) }\end{array}$ & $\begin{array}{c}\delta^{13} \mathrm{C}_{\mathrm{CO} 2 \text { fluid }} \\
(\% \text { of })\end{array}$ & $\begin{array}{c}\delta^{13} \mathrm{C}_{\mathrm{CO} 2 \text { fluid }} \\
(\% \mathrm{oo}) \mathrm{g})\end{array}$ \\
\hline 1 & SpFA2v & Cal-Arg & 2.16 & -15.07 & 15.37 & 113.47 & 116.99 & 104.09 & -1.01 & -1.21 & -1.76 \\
\hline 2 & SpFA6.3v & Cal-Arg & -0.12 & -14.98 & 15.47 & 112.68 & 116.00 & 103.36 & -3.53 & -3.35 & -4.08 \\
\hline 3 & SpFA7av & Cal-Arg & 0.96 & -12.17 & 18.36 & 89.52 & 88.51 & 81.88 & -3.89 & -3.96 & -4.42 \\
\hline 4 & SpFA9v & Cal-Arg & -3.66 & -14.94 & 15.51 & 112.33 & 115.57 & 103.04 & -7.09 & -6.91 & -7.64 \\
\hline 5 & SpFA38v & Cal-Arg & -0.36 & -14.41 & 16.05 & 107.74 & 109.92 & 98.80 & -4.06 & -3.93 & -4.60 \\
\hline 6 & SpFA39v & Cal-Arg & -3.17 & -15.41 & 15.02 & 116.49 & 120.79 & 106.88 & -6.37 & -6.14 & -6.92 \\
\hline 7 & SpFA40v & Cal-Arg & -3.11 & -15.06 & 15.38 & 113.38 & 116.88 & 104.01 & -6.48 & -6.29 & -7.03 \\
\hline 8 & SpFA42v & Cal-Arg & 0.22 & -15.02 & 15.42 & 113.03 & 116.44 & 103.69 & -3.17 & -2.98 & -3.72 \\
\hline 9 & SpFA44v & Cal-Arg & -0.48 & -14.32 & 16.15 & 106.98 & 108.99 & 98.09 & -4.22 & -4.11 & -4.77 \\
\hline 10 & SpFA45v & Cal-Arg & -2.79 & -14.95 & 15.50 & 112.42 & 115.68 & 103.12 & -6.22 & -6.04 & -6.76 \\
\hline 11 & SpFA28v & Cal & 0.45 & -14.32 & 16.15 & 106.98 & 108.99 & 98.09 & -3.29 & -3.18 & -3.84 \\
\hline 12 & SpFA29v & Cal & 0.32 & -14.09 & 16.38 & 105.03 & 106.63 & 96.28 & -3.54 & -3.44 & -4.08 \\
\hline 13 & SpFA29.1v & Cal & -0.4 & -14.37 & 16.09 & 107.40 & 109.51 & 98.48 & -4.12 & -4.00 & -4.66 \\
\hline 14 & SpFA30v & Cal & -0.81 & -11.39 & 19.17 & 83.57 & 81.86 & 76.35 & -6.07 & -6.19 & -6.59 \\
\hline 15 & SpFA10v & Cal-Arg & 1.12 & -11.97 & 18.57 & 87.97 & 86.77 & 80.45 & -3.83 & -3.92 & -4.36 \\
\hline Average & & & & & & 105.93 & 107.97 & 90.17 & $-4,39$ & -4.30 & -4.90 \\
\hline
\end{tabular}

Note: n.d., non-detected. ${ }^{\mathrm{a}}{ }^{18} \mathrm{O}_{\mathrm{V}-\mathrm{PDB}}$ converted in $\delta^{18} \mathrm{O}_{\mathrm{V}-S M O W}$ using the equation: $\delta^{18} \mathrm{O}_{\mathrm{V}-S M O W}=1.03091^{*} \delta^{18} \mathrm{O}_{\mathrm{V}-\mathrm{PDB}}+30.91$ from [68]. ${ }^{\mathrm{b}}$ Temperature calculated using the equation proposed by [81]. ${ }^{\mathrm{c}}$ Temperature calculated using the equation proposed by [81]; [82]. ${ }^{\mathrm{d}}$ Temperature calculated using the equation proposed by [83]. ${ }^{\mathrm{e}} \delta^{13} \mathrm{C}_{\mathrm{CO} 2}$ fluid calculated using the equation proposed by [87] considering ${ }^{b} .{ }^{f} \delta^{13} \mathrm{C}_{\mathrm{CO}}$ fluid calculated using the equation proposed by [87] considering ${ }^{\mathrm{c}}$. $\mathrm{g}^{13} \mathrm{C}_{\mathrm{CO} 2}$ fluid calculated using the equation proposed by [87] considering ${ }^{d}$.

Table 7. $\mathrm{C}$ and $\mathrm{O}$ isotope data and calculated fluid isotopic composition for the dolomite in serpentinites from the Pietrapica site.

\begin{tabular}{|c|c|c|c|c|c|c|c|c|c|}
\hline Sample No. & Sample Code & $\begin{array}{c}\text { Carbonate } \\
\text { Phase }\end{array}$ & $\delta^{13} C_{\text {PDB }}(\% o)$ & $\delta^{18} \mathrm{O}_{\mathrm{PDB}}(\%$ o $)$ & $\begin{array}{c}\mathcal{\delta}^{18} \mathrm{O}_{\text {SMOW }} \\
(\%)^{\mathrm{a})}\end{array}$ & $\mathrm{T}^{\circ} \mathrm{C}^{\mathrm{b})}$ & $\mathrm{T}^{\circ} \mathrm{C}^{\mathrm{c})}$ & $\begin{array}{c}\delta^{13} \mathrm{C}_{\mathrm{CO} 2 \text { fluid }} \\
(\% \text { o })\end{array}$ & $\begin{array}{c}\delta^{13} \mathrm{C}_{\mathrm{CO} 2 \text { fluid }} \\
(\% \text { o) }\end{array}$ \\
\hline 1 & SpPP33v & Dol-Mg-Cal & -2.69 & -9.12 & 21.51 & 105.04 & 83.77 & -6.97 & -8.40 \\
\hline 2 & SpPP33.1v & Dol-Mg-Cal & -3.37 & -9.17 & 21.45 & 105.57 & 84.15 & -7.61 & -9.05 \\
\hline 3 & SpPP33Dv & Dol-Ank & -2.7 & -9.34 & 21.28 & 107.39 & 85.43 & -6.83 & -8.28 \\
\hline 4 & SpPP33D.1v & Dol-Ank & -3.6 & -9.4 & 21.22 & 108.04 & 85.88 & -7.69 & -9.15 \\
\hline 5 & SpPP35v & Dol-Cal & -1.79 & -9.49 & 21.13 & 109.02 & 86.57 & -5.83 & -7.29 \\
\hline 6 & SpPP35.1v & Dol-Cal & -2.26 & -9.57 & 21.04 & 109.90 & 87.18 & -6.24 & -7.72 \\
\hline Average & & & & & & 107.49 & 85.50 & -6.86 & -8.32 \\
\hline
\end{tabular}

Note: $n . d=$ non-detected. ${ }^{a} \delta^{18} \mathrm{O}_{\mathrm{V}-\mathrm{PDB}}$ converted in $\delta^{18} \mathrm{O}_{\mathrm{V}-S M O W}$ using the equation: $\delta^{18} \mathrm{O}_{\mathrm{V}-\mathrm{SMOW}}=1.03091^{*} \delta^{18} \mathrm{O}_{\mathrm{V}-\mathrm{PDB}}+30.91$ from [68]. ${ }^{\mathrm{b}}$ Temperature calculated using the equation proposed by [84]. ${ }^{\mathrm{c}}$ Temperature calculated using the equation proposed by [85]. ${ }^{\mathrm{d}} \delta^{13} \mathrm{C}_{\mathrm{CO} 2}$ fluid calculated using the equation proposed by [85] considering ${ }^{\mathrm{b}}$. ${ }^{\mathrm{s}} \delta^{13} \mathrm{C}_{\mathrm{CO} 2}$ fluid calculated using the equation proposed by [85] considering ${ }^{c}$. 
Similarly, for the vein samples of SpPP, the equilibrium temperature was inferred in the range between 105 and $110{ }^{\circ} \mathrm{C}\left(\Delta^{18} \mathrm{O}_{\text {dolomite }}\right.$ from [84]) and between 83 and $87^{\circ} \mathrm{C}\left(\Delta^{18} \mathrm{O}_{\text {dolomite }}\right.$ from [85]).

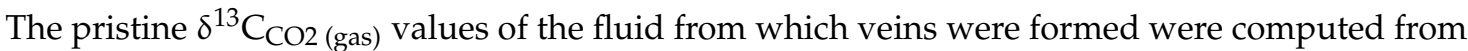
the $\delta^{13} \mathrm{C}_{\mathrm{CaCO} 3}$ values and the calculated carbonate deposition temperature for each carbonate sample. We have assumed the achievement of the isotope equilibrium between fluid $\mathrm{CO}_{2}$ and a carbonate mineralogical phase (calcite in the SpFA veins and dolomite in SpPP veins) and applied the following equation:

$$
\delta^{13} \mathrm{C}_{\mathrm{CO} 2}=\delta^{13} \mathrm{C}_{\text {carb.min }} \Delta_{\text {carb.min-CO2 }}
$$

where $\delta^{13} \mathrm{C}_{\text {carb.min }}$ is the isotope composition of the considered carbonate mineralogical phase, and $\Delta_{\text {carb.min-CO2 }}$ is the equilibrium fractionation factor for carbon between the carbonate mineral and $\mathrm{CO}_{2}$ and calcite [87] and between $\mathrm{CO}_{2}$ and dolomite [85] (Tables 6 and 7). Based on the equilibrium temperature estimated using the fractionation factors computed by [81], [82], and [83], the average $\delta^{13} \mathrm{C}_{\mathrm{CO} 2}$ values of SpFA veins is in the range from $-4.36 \%$ o to $-5.02 \%$. Slightly more negative average $\delta^{13} \mathrm{C}_{\mathrm{CO} 2}$ values (from $-6.86 \%$ o to $-8.32 \%$ ) were obtained for SpPP veins if we consider the equilibrium temperature estimated following [84] and [85], respectively.

\subsection{Fluid Inclusions Hosted by Quartz in Sppp Veins}

Most of the fluid inclusions in the quartz are arranged along lines of crystal growth, and thus, they are considered as primary and/or pseudosecondary fluids according to the criteria defined by [88] (Figure 7a). Some fluid inclusions occur along secondary trails and necking down is occasionally seen. In Table 8, the primary or secondary origin of each fluid inclusion is indicated. When possible, fluid inclusion assemblages [89] have been analyzed. Two major types of fluid inclusions were recognized at room temperature: predominantly all-liquid fluid inclusions (single-phase inclusions: $\mathrm{L}_{\mathrm{H} 2 \mathrm{O}}$ ) and in less amount liquid-vapor inclusions (biphasic inclusions: $\mathrm{L}_{\mathrm{H} 2 \mathrm{O}}+\mathrm{V}_{\mathrm{H} 2 \mathrm{O}}$ ) (Figure $7 \mathrm{~b}$ ). All-liquid fluid inclusions usually nucleated a small gas bubble with little heating (around $50{ }^{\circ} \mathrm{C}$ ) (Figure $7 \mathrm{c}$ ). This means that they are in a metastable state out of their stability field. Only the smallest ones (usually $<5 \mu \mathrm{m}$ ) remain all-liquid after heating. These ones cannot be used for microthermometric studies because a bubble is required for temperature determinations.
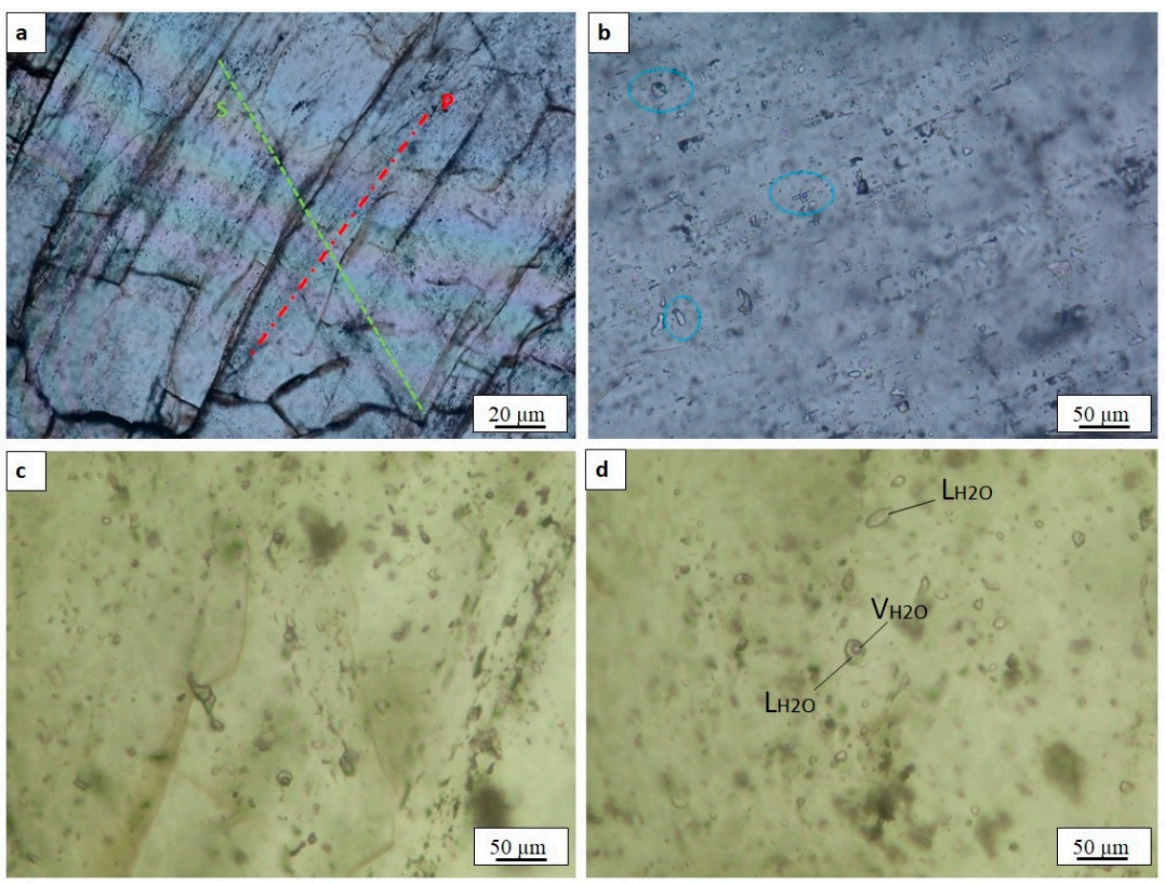

Figure 7. Photomicrographs of fluid inclusions in quartz of veins from SpPP. Primary (red dotted line) and secondary (green dotted line) fluid inclusions (a); fluid inclusions at room temperature (b) and at $\sim 50^{\circ} \mathrm{C}(\mathbf{c})$; monophasic $\left(\mathrm{L}_{\mathrm{H} 2 \mathrm{O}}\right)$ and biphasic fluid inclusions $\left(\mathrm{L}_{\mathrm{H} 2 \mathrm{O}}+\mathrm{V}_{\mathrm{H} 2 \mathrm{O}}\right)(\mathbf{d})$. 
Table 8. Microthermometric data of fluid inclusions in quartz crystals in a quartz-carbonate vein from the Pietrapica quarry (SpPP).

\begin{tabular}{|c|c|c|c|c|c|c|c|c|}
\hline IF & FIA & Origin & $T_{\mathrm{h}}\left({ }^{\circ} \mathrm{C}\right)$ & $T_{\mathrm{m}}\left(\right.$ Ice) $\left({ }^{\circ} \mathrm{C}\right)$ & $T_{\mathrm{n}}\left({ }^{\circ} \mathrm{C}\right)$ & $\begin{array}{l}\text { Size } \\
(\mu \mathrm{m})\end{array}$ & $\mathrm{L}: \mathrm{V}$ & $\begin{array}{c}\text { Salinity } \\
\text { (NaCl Mass \%) }\end{array}$ \\
\hline SpPP36.1_1v & n.d. & $\mathrm{P}$ & $198-200$ & -0.8 & -30 & 18 & $80 / 20$ & 1.4 \\
\hline SpPP36.1_2v & 1 & $S$ & $121-124$ & + & -33 & 8 & $95 / 5$ & n.d. \\
\hline SpPP36.1_3v & 1 & $\mathrm{~S}$ & $125-130$ & -0.8 & 32 & 6 & $95 / 5$ & 1.4 \\
\hline SpPP36.1_4v & 2 & $\mathrm{P}$ & n.d. & -0.6 & -36 & 50 & $90 / 10$ & 1.05 \\
\hline SpPP36.1_5v & 2 & $\mathrm{~S}$ & $120-125$ & + & -40 & 7 & $95 / 5$ & n.d. \\
\hline SpPP36.1_6v & n.d. & $\mathrm{S}$ & 185-188 & -0.3 & -34 & 4 & $90 / 10$ & 0.53 \\
\hline SpPP36.1A_7v & n.d. & n.d. & n.d. & + & -30 & n.d. & n.d. & n.d. \\
\hline SpPP36.1A_8v & n.d. & n.d. & $112-125$ & + & -30 & 8 & $95 / 5$ & n.d. \\
\hline SpPP36.1A_10v & n.d. & n.d. & n.d. & + & -28 & 40 & $85 / 15$ & n.d. \\
\hline SpPP36.1A_11v & n.d. & n.d. & 190-196 & -0.8 & -29 & 10 & $90 / 10$ & 1.4 \\
\hline SpPP36.1A_1v & n.d. & n.d. & n.d. & + & -29 & 6 & $95 / 5$ & n.d. \\
\hline SpPP34.1_13v & 3 & $\mathrm{P}$ & $>200$ & $(-1.9)(-1.6)$ & -30 & 17 & $70 / 30$ & $3.23-2.74$ \\
\hline SpPP34.1_14v & 3 & $\mathrm{P}$ & $138-140$ & + & -31.3 & 7 & $95 / 5$ & n.d. \\
\hline SpPP34.1_15v & 4 & S & $118-120$ & + & -32.5 & 4 & $95 / 5$ & n.d. \\
\hline SpPP34.1_16v & 5 & $\mathrm{P}$ & $>200$ & -1.9 & -32 & 6 & $90 / 5$ & 3.23 \\
\hline SpPP34.1_17v & 6 & S & $>200$ & -1.8 & n.d. & 4 & $90 / 10$ & 3.06 \\
\hline SpPP34.1_18v & 6 & S & $118-120$ & -0.8 & -31 & 10 & $95 / 5$ & 1.4 \\
\hline SpPP34.1_19v & 7 & $S$ & n.d. & + & -29.7 & 7 & $95 / 5$ & n.d. \\
\hline SpPP34.1_20v & n.d. & $\mathrm{P}$ & $105-110$ & -0.7 & -30.8 & 11 & $90 / 10$ & 1.23 \\
\hline SpPP34.1_21v & 8 & $S$ & n.d. & + & -34.8 & 6 & $95 / 5$ & n.d. \\
\hline SpPP34.4_22v & 9 & $\mathrm{P}$ & n.d. & $(-0.9)(-0.7)$ & -31 & 11 & $70 / 30$ & $1.57-1.23$ \\
\hline SpPP34.4_23v & 9 & $\mathrm{P}$ & n.d. & -0.9 & -30 & 10 & $90 / 10$ & 1.57 \\
\hline SpPP34.4_24v & 8 & $S$ & n.d. & & -28 & 10 & n.d. & n.d. \\
\hline SpPP34.4_25v & 8 & S & n.d. & -0.3 & -29 & 5 & $95 / 5$ & 0.53 \\
\hline SpPP34.4_26v & 10 & $\mathrm{P}$ & n.d. & + & -30.8 & 7 & $95 / 5$ & n.d. \\
\hline SpPP34.4_27v & 10 & $\mathrm{P}$ & $280-288$ & $(-0.7)(-0.5)$ & -33.6 & 8 & $90 / 10$ & $1.23-0.88$ \\
\hline SpPP34.4_28v & 11 & $\mathrm{~S}$ & $>200$ & -0.7 & -34.2 & 5 & $90 / 10$ & 1.23 \\
\hline SpPP34.4_29v & 11 & $S$ & 120.8 & + & -31.8 & 4 & $95 / 5$ & n.d. \\
\hline SpPP34.4_30v & 12 & $S$ & $210-216$ & -0.7 & -34.2 & 7 & $90 / 10$ & 1.23 \\
\hline SpPP34.4_31v & 13 & $S$ & $288-293$ & -0.4 & -34.8 & 5 & $50 / 50$ & 0.71 \\
\hline SpPP34.4_32v & 14 & $\mathrm{P}$ & 93 & -0.6 & -34.2 & 8 & $90 / 10$ & 1.05 \\
\hline SpPP34.4_33v & n.d. & $\mathrm{P}$ & $120-130$ & n.d. & n.d. & 5 & $95 / 5$ & n.d. \\
\hline SpPP34.4_34v & n.d. & $\mathrm{P}$ & 330 & n.d. & n.d. & 7 & $10 / 90$ & n.d. \\
\hline SpPP34.4_35v & n.d. & $\mathrm{P}$ & $225-235$ & n.d. & n.d. & 4 & $50 / 50$ & n.d. \\
\hline SpPP34.4_36v & n.d. & $\mathrm{P}$ & $>395$ & n.d. & n.d. & n.d. & $10 / 90$ & n.d. \\
\hline SpPP34.4_37v & n.d. & $\mathrm{P}$ & 335 & n.d. & n.d. & n.d. & $10 / 90$ & n.d. \\
\hline
\end{tabular}

Note: n.d. $=$ not-detected; $+=$ positive value; FIA $=$ fluid inclusions association; $T_{\mathrm{h}}\left({ }^{\circ} \mathrm{C}\right)=$ homogenization temperatures; $T_{\mathrm{m}}(\mathrm{Ice})\left({ }^{\circ} \mathrm{C}\right)=$ temperatures of final ice-melting; $T_{\mathrm{n}}\left({ }^{\circ} \mathrm{C}\right)=$ nucleation temperature; $\mathrm{L}: \mathrm{V}=$ liquid vapor ratio; Salinity $=$ wt. $\% \mathrm{NaCl}$ calculated from $T_{\mathrm{m}}$ (Ice).

Collectively, the two types of fluid inclusions have rounded and sub-rounded shapes and exhibit a relatively wide range of liquid/vapor volume ratios (some with ratios around 95:5 and others with ratios between $50: 50$ and 90:10), indicating a heterogeneous entrapment.

We did not find any evidence for the presence of $\mathrm{CO}_{2}$ - and $\mathrm{CH}_{4}$-phases $\left(\mathrm{Tm}=-56.6\right.$ and $-147.0^{\circ} \mathrm{C}$ for $\mathrm{CO}_{2}$ - and $\mathrm{CH}_{4}$, respectively). In fact, during the cooling phase, we went down until liquid nitrogen temperatures and no melting process different than the melting of ice was observed. This means that the gas is likely water vapor (Figure 7d). However, the presence of another gas different from water vapor cannot be discarded. If present, this gas would have very low density $\left(\mathrm{H}_{2}\right.$ or $\left.\mathrm{He}\right)$, so that would be undetectable by microthermometry. The temperatures of final ice-melting $\mathrm{Tm}$ (Ice) values range from -0.3 to $-1.9^{\circ} \mathrm{C}$. Many inclusions present positive ice-melting temperatures, which means that they are under high pressure out of their stability field. Temperatures of the first melting (eutectic temperature, $\mathrm{Te}$ ) were observed around $-30{ }^{\circ} \mathrm{C}$, which is the metastable temperature of the $\mathrm{H}_{2} \mathrm{O}+$ $\mathrm{NaCl}$ system. 
Based on the Tm(Ice) and taking into account the $\mathrm{H}_{2} \mathrm{O}+\mathrm{NaCl}$ system, the biphasic inclusions are found to be of low salinity, between 0.53 and $3.23 \mathrm{NaCl}$ mass \% equivalent (using equation by [71]). All the biphasic inclusions homogenize to liquid, with the final homogenization temperature (Th) present in two different ranges of temperature: the first one from 93 to $140{ }^{\circ} \mathrm{C}$ and the second one from 185 to $335^{\circ} \mathrm{C}$.

\section{Discussions}

\subsection{Mineral Assemblage}

As reported by previous studies, the mineral assemblages and texture of serpentinites of the Frido Unit show evidence of ocean floor metamorphism [54,55]. In addition to minerals typical of worldwide serpentinites, including serpentine minerals, amphiboles, pyroxene, chlorite, titanite, and magnetite, the Frido Unit serpentinites are characterized by talc and hydro-garnet. In particular, the presence of garnet in the serpentinitic rocks from the studied area has been documented here for the first time.

Hydro-andradite, containing variable amounts of $\mathrm{TiO}_{2}$ (0.75 to $3.60 \mathrm{wt} \%$, Table 3), occurs in several mineral assemblages of serpentinites, among which the "serpentine + diopside + magnetite" association is one of the most common. Hydro-garnet is stable in these rocks over a wide range of oxygen fugacities and Ca activities, and its stability is controlled by the following reaction [90]:

$$
\begin{gathered}
3 \mathrm{Ca}_{3} \mathrm{Fe}_{2} \mathrm{Si}_{3} \mathrm{O}_{12}+9 \mathrm{SiO}_{2}+\mathrm{Fe}_{2} \mathrm{O}_{3}=9 \mathrm{CaFeSi}_{2} \mathrm{O}_{6}+2 \mathrm{O}_{2} \\
\text { andradite }+ \text { silica }+ \text { magnetite }=\text { pyroxene }+ \text { fluid }
\end{gathered}
$$

The titanian hydro-andradite may form in both magmatic and hydrothermal systems. According to [91], in fact, the presence of such a mineral has been documented in rocks associated with silica undersaturated magmatic systems (carbonatites, kimberlites, alkaline intrusions) as well as with hydrothermally alterated oceanic lithosphere (for example the Sanbagawa metamorphic complex, the mid-Atlantic ridge, the Nagaland ophiolite belt), testifying intermediate to low (150 to $\left.300{ }^{\circ} \mathrm{C}\right)$ temperature fluids with low $\mathrm{SiO}_{2}$ activity.

The hydrothermal activity is also thought of as responsible for the talc occurrence in the studied serpentinites. Frost et al. [90] stated that hydrothermal fluids in equilibrium with basic rocks may have high enough silica activity to alter serpentine to talc following the reactions below [16]:

$$
\begin{aligned}
& \mathrm{Mg}_{3} \mathrm{Si}_{2} \mathrm{O}_{5}(\mathrm{OH})_{4}+2 \mathrm{SiO}_{2(\mathrm{aq})} \rightarrow \mathrm{Mg}_{3} \mathrm{Si}_{4} \mathrm{O}_{10}(\mathrm{OH})_{2}+\mathrm{H}_{2} \mathrm{O} \\
& \text { serpentine }+ \text { silica-saturated fluid } \rightarrow \text { talc }+ \text { aqueous fluid }
\end{aligned}
$$

and/or

$$
\begin{gathered}
2 \mathrm{Mg}_{3} \mathrm{Si}_{2} \mathrm{O}_{5}(\mathrm{OH})_{4}+3 \mathrm{CO}_{2}+3 \mathrm{CaCO}_{3} \rightarrow \mathrm{Mg}_{3} \mathrm{Si}_{4} \mathrm{O}_{10}(\mathrm{OH})_{2}+3 \mathrm{CaMg}\left(\mathrm{CO}_{3}\right)_{2}+3 \mathrm{H}_{2} \mathrm{O} \\
\text { serpentine }+\mathrm{CO}_{2} \text {-rich fluid }+ \text { calcite } \rightarrow \text { talc }+ \text { dolomite }+ \text { aqueous fluid }
\end{gathered}
$$

Based on the mineralogical composition of studied samples, as also confirmed by the petrographic observations (Figure $4 \mathrm{~d}$ ), the above reactions mirror the rock-fluid interaction processes that have involved samples from the Pietrapica site only, in which talc-rich domains and dolomite have been detected. However, in such samples along with talc and carbonate minerals, quartz is present as well. According to Moore and Rymer [92], a large amount of dissolved silica may be supplied to serpentinite rocks during the hydrothermal alteration of serpentine. Therefore, the quartz in the studied samples could represent the result of direct precipitation from a silica-rich fluid derived from the breakdown of serpentinite-forming silicates (serpentine).

The mode of occurrence of quartz in the SpPP veins suggests a further consideration of the chemistry of the mineralizing fluid in the Pietrapica area. The petrographical study of veins, in fact, 
has shown that intergrowth structures characterize quartz and dolomite in those samples, and this is consistent with the hypothesis of a contemporaneous formation of silicate and carbonate phases from the same source fluid. As a consequence, the chemical features of fluid inclusions in the quartz can be assumed as representative of the chemistry of the whole mineralizing fluid.

As for veins from the Fosso Arcangelo site, no hypothesis can be made on the basis of their mineralogical composition only. These veins are dominated by calcite as the principal carbonate phase. Calcite is a common mineral because it may form in a great variety of geological settings. It represents an important rock-forming mineral in sedimentary rocks, can be an essential component of metamorphic and igneous rocks, and is common in hydrothermal environments [93]. In particular, in geothermal systems, the calcite formation is chiefly favored by boiling, dilution, and condensation processes that control its occurrence, distribution, and stable isotope composition [94]. Further, during serpentinization, the mineralogical and geochemical processes transforming the oceanic lithosphere usually produce Ca-rich fluids that can migrate in the hydrothermal system and promote carbonates precipitation (mainly calcite) as serpentinite matrix and/or infill of veins and veinlets $[35,95,96]$. In the SpFA veins, calcite locally is in association with serpentine and amphibole crystals. Habitus of crystals and the lack of a preferential orientation of these silicates allow us to suppose that they were englobed into the hydrothermal fluid during its migration through the serpentinite host rocks. The lobate contacts between serpentine and/or amphibole crystals and calcite support this hypothesis.

\subsection{Temperature of Precipitation, Fluid Composition, and Sources}

Stable isotope (carbon and oxygen) geochemistry provides relevant constraints about metasomatic processes involving carbonates [97-101]. As previously stated, based on $\delta^{18} \mathrm{O}$ data and assuming the water/mineral isotope equilibrium, equilibrium temperature fluctuates in a narrow range for both the vein samples $\left(T=80-120^{\circ} \mathrm{C}\right.$ for SpFA and $T=80-110^{\circ} \mathrm{C}$ for SpPP). Nonetheless, the veins belonging to different outcrops show different prevalent carbonate minerals (calcite in SpFA and dolomite for SpPP) and occurred under similar thermal regimes. The lack of a positive correlation between $\delta^{13} \mathrm{C}_{\mathrm{CaCO} 3}$ and $\delta^{18} \mathrm{O}_{\mathrm{CaCO} 3}$ (Figure 8) seems to indicate that the depositional temperature controls, exclusively, the isotope signature of the carbonate veins. Therefore, the computed $\delta^{13} \mathrm{C}_{\mathrm{CO} 2 \text {-gas }}$ values are representative of the original $\mathrm{CO}_{2}$ supplied during vein formation.

The average fluid $\delta^{13} \mathrm{C}_{\mathrm{CO} 2}$ inferred from the fluid-carbonates isotope equilibrium range from $-4.36 \%$ o to $-5.32 \%$ in the veins of the SpFA and from $-6.86 \%$ o to $-8.32 \%$ in the veins of the SpPP.

The range of the $\mathrm{C}$-isotope composition of both vein groups is slightly more negative than that of seawater carbonates $\left(\delta^{13} \mathrm{C}_{\mathrm{CO} 2}\right.$ around $0 \%$ ), whereas it lies fully within the range of $\delta^{13} \mathrm{C}_{\mathrm{CO} 2}$ values typically associated to mantle carbon $\left(-8 \%\right.$ o $<\delta^{13} \mathrm{C}_{\mathrm{CO} 2}<-4 \%$; [102-104]. Degassing of $\mathrm{CO}_{2}-$ rich fluids during their rising from the underlying lithospheric mantle might lead to the precipitation of calcite [105]. Thus, the inferred $\delta^{13} \mathrm{C}_{\mathrm{CO} 2}$ values are consistent with a magmatic $\mathrm{CO}_{2}$ component in the hydrothermal fluids.

However, the available data do not allow us to rule out that carbon in these veins might derive from other sources than the mantle. In fact, the mixing between fluids having different isotope carbon isotope composition (e.g., seawater and carbon derived from organic rich sediments or from the oxidation of methane having $\delta^{13} \mathrm{C}_{\mathrm{CO} 2}<-15 \%$ o) would reproduce $\delta^{13} \mathrm{C}_{\mathrm{CO} 2}$ values in the same range to those inferred for the SpFA and SpPP veins.

Alternatively, decarbonation of marine sediments having a typical isotope signature (e.g., $\delta^{13} \mathrm{C}$ close to $0 \%$, [106] would produce a lowering of $\delta^{13} \mathrm{C}$, with or without change in $\delta^{18} \mathrm{O}$, and an $\mathrm{O}$ shift can be observed only when decarbonation is driven by infiltration by externally derived $\mathrm{H}_{2} \mathrm{O}$-rich fluids [34]. 


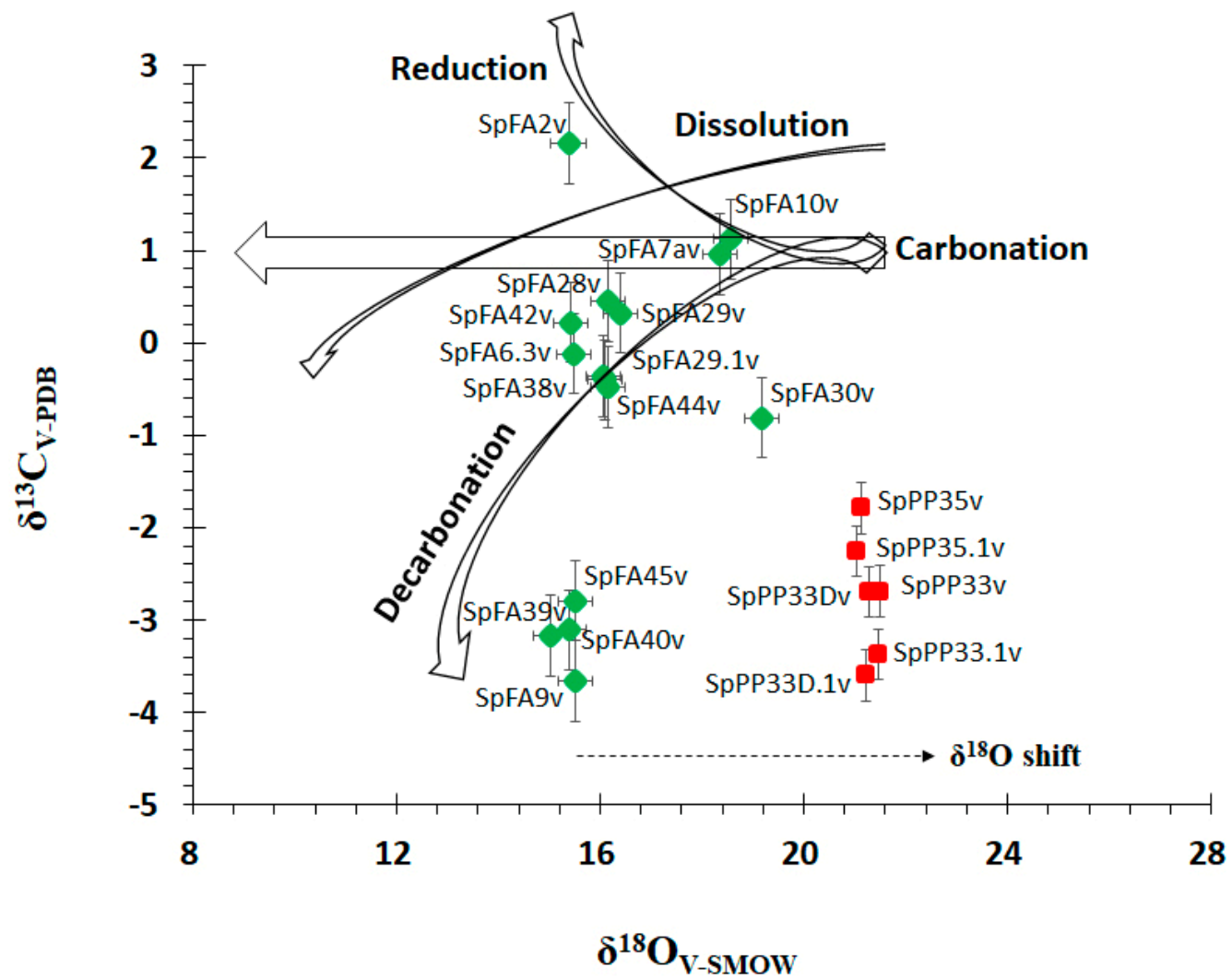

Figure 8. $\delta^{13} \mathrm{C}_{\mathrm{V}-\mathrm{PDB}}$ vs. $\delta^{18} \mathrm{O}_{\mathrm{V}-\mathrm{SMOW}}$ plot of calcite/dolomite in the serpentinites from the Fosso Arcangelo site (SpFA = green diamonds) and Pietrapica quarry (SpPP = red squares). Geochemical trends of carbonates affected by dissolutions [99], carbonate reduction [101], and carbonation and decarbonation [35] are also shown.

As shown in Figure 8, samples from the two studied sites fall in different fields of the $\delta^{18} \mathrm{O}$ vs. $\delta^{13} \mathrm{C}$ diagram. In detail, the SpFA veins overlap the pathway typical of the decarbonation process as suggested by [35] and references therein. Such a process likely developed at depth in the crust in a "closed system" wherein no external fluid supply can occur.

On the contrary, for the SpPP site, vein samples are characterized by an oxygen isotope shift toward more positive $\delta^{18} \mathrm{O}$ values. Moreover, significant differences in the mineralogical assemblage were also found in SpFA and SpPP veins, being calcite prevalent in the former veins and dolomite and quartz in the latter ones. Intergrowth structures between dolomite and quartz crystals suggest the hypothesis of a contemporaneous formation of silicate and carbonate phases in the SpPP veins.

Data of fluid inclusions in the quartz (in SpPP veins) show abundant aqueous $\left(\mathrm{L}_{\mathrm{H} 2 \mathrm{O}}-\mathrm{V}_{\mathrm{H} 2 \mathrm{O}}\right.$ ) and low salinity features (between 0.53 and $3.23 \mathrm{NaCl}$ mass \% equivalent). Further, the decrease of salinity associated with the decrease of homogenization temperature $\left(335-185^{\circ} \mathrm{C}\right.$ and $\left.140-93{ }^{\circ} \mathrm{C}\right)$ may be related to a large infiltration of shallow, diluted, and fresh waters that also led to a progressive cooling of the hydrothermal system. Therefore, it seems that SpPP veins are consistent with a crystallization in an open-system at shallower crustal conditions.

Accordingly, all these features, even not well-constrained would suggest the hypothesis that SpFA and SpPP veins were deposited under different boundary conditions (e.g., temperature), and/or from parental fluids having different chemical composition.

Further detailed investigations on the fluid inclusions composition as well as on the oxygen isotopes of the silicate are requested to clarify the formation process of these veins and to fully understand the role and the compositions of the parental fluid(s). 


\section{Conclusions}

Our study demonstrates that, in serpentinites of the Frido Unit (southern Apennines), different types of veins occur recording fluid production and migration in the accretionary wedge of the southern Apennines. We envisage at least two formation episodes for veins that crosscut serpentines from the studied sites (Fosso Arcangelo and Pietrapica) within the same thermal system (Figure 9a). We have identified a first vein group formed by decarbonation of serpentinites in a closed hydrothermal system that generated $\mathrm{Ca}$ - and $\mathrm{CO}_{2}$-rich fluids from which carbonates of the Fosso Arcangelo veins derive. Even if not well-constrained, such fluids could migrate toward shallower depths, modifying their composition by interaction with serpentinite host rocks. Then, fluid migration ended in an open hydrothermal system where mixing between deep Si and Mg enriched fluids and externally derived $\mathrm{H}_{2} \mathrm{O}$-rich fluids occurred, promoting the quartz-carbonate vein formation in the Pietrapica serpentinites. Subsequently, serpentinite slices and associated veins were involved by tectonic activity related to the formation of the Liguride Complex and finally exhumed (Figure 9b).

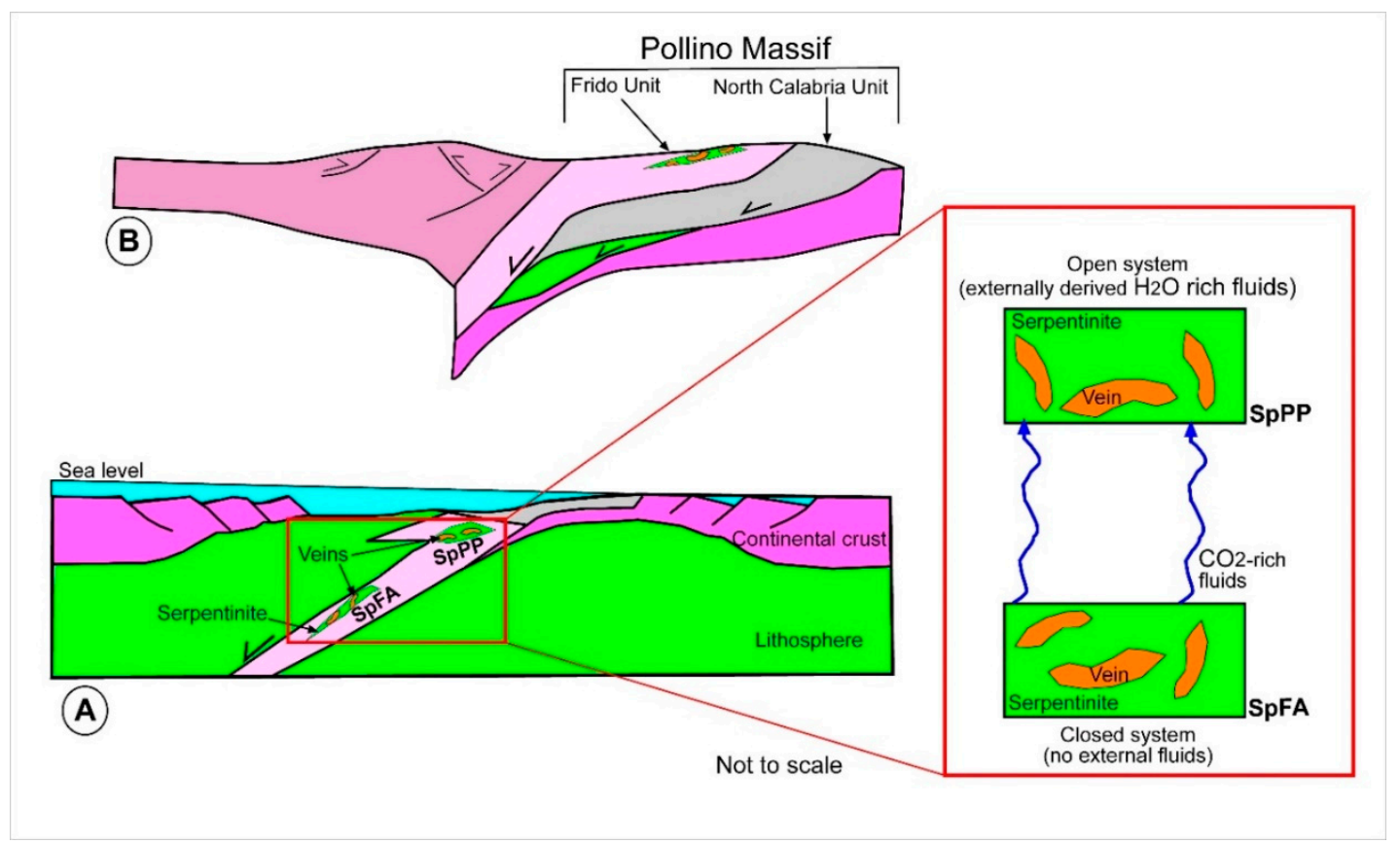

Figure 9. Bi-dimensional sketch displaying the tectonic evolution of the Liguride Complex. Inset (a) shows the position of serpentinites and cutting veins of Fosso Arcangelo (SpFA) and Pietrapica (SpPP) sites in the subduction zone. In the red box is shown a scheme illustrating the different hydrothermal systems of both studied sites along with the pathway of migrating fluids (not in scale). Inset (b) displays the actual position of studied rocks in the accretionary wedge of the Pollino Massif. See the text further details.

As a consequence, our study contributes to a better understanding of processes leading to mobilization, fractionation, and redistribution of chemical elements within subduction zones, with particular attention to the southern Apennines.

Author Contributions: Conceptualization, G.R. and G.M.; Methodology, M.C.D.; Validation, P.C., F.G. and M.P.; Formal Analysis, M.C.D., F.G., M.P.; Investigation, M.C.D., S.L., R.S.; Data Curation, M.C.D. and S.L.; Writing-Original Draft Preparation, M.C.D; Writing-Review \& Editing, G.R. and R.S.; Supervision, G.M.; Funding Acquisition, G.M. and P.C. All authors have read and agreed to the published version of the manuscript.

Funding: This work was partly funded by Mongelli G. grant (RIL2016) and by the project CGL2016-78560-P of the Spanish Ministerio de Economía y Competitividad.

Acknowledgments: The authors wish to thank González Acebrón Laura (Departamento de Estratigrafía, Universidad Complutense, Madrid, España) for fluid inclusions studies. 
Conflicts of Interest: The authors declare no conflict of interest.

\section{References}

1. Alt, J.C.; Shanks III, W.C.; Crispini, L.; Gaggero, L.; Schwarzenbach, E.M.; Früh-Green, G.L.; Bernasconi, S.M. Uptake of carbon and sulphur during seafloor serpentinization and the effects of subduction metamorphism in Ligurian peridotites. Chem. Geol. 2012, 322, 268-277. [CrossRef]

2. Tucholke, B.E.; Lin, J. A geological model for the structure of ridge segments in slow spreading ocean crust. J. Geophys. Res. Space Phys. 1994, 99, 11937-11958. [CrossRef]

3. Cannat, M.; Mevel, C.; Maia, M.; Deplus, C.; Durand, C.; Gente, P.; Agrinier, P.; Belarouchi, A.; Dubuisson, G.; Humler, E.; et al. Thin crust, ultramafic exposures, and rugged faulting patterns at the Mid-Atlantic Ridge $\left(22^{\circ}-24^{\circ} \mathrm{N}\right)$. Geology 1995, 23, 49-52. [CrossRef]

4. Cannat, M.; Fontaine, F.; Escartin, J. Serpentinization and associated hydrogen and methane fluxes at slow spreading ridges. In Sea Ice; American Geophysical Union (AGU): Washington, DC, USA, 2010; Vol. 188, pp. 241-264.

5. Cann, J.R.; Blackman, D.K.; Smith, D.K.; McAllister, E.; Janssen, B.; Mello, S.; Avgerinos, E.; Pascoe, A.R.; Escartin, J.; Cann, D.K.B.J.R. Corrugated slip surfaces formed at ridge-transform intersections on the Mid-Atlantic Ridge. Nature 1997, 385, 329-332. [CrossRef]

6. Blackman, D.K.; Janssen, B.; Smith, D.K.; Cann, J.R. Origin of extensional core complexes: Evidence from the Mid-Atlantic Ridge at Atlantis Fracture Zone. J. Geophys. Res. Space Phys. 1998, 103, 21315-21333. [CrossRef]

7. Smith, D.K.; Cann, J.R.; Escartín, J. Widespread active detachment faulting and core complex formation near $13^{\circ} \mathrm{N}$ on the Mid-Atlantic Ridge. Nature 2006, 442, 440-443. [CrossRef]

8. Schwarzenbach, E.M.; Früh-Green, G.L.; Bernasconi, S.M.; Alt, J.C.; Iii, W.C.S.; Gaggero, L.; Crispini, L. Sulfur geochemistry of peridotite-hosted hydrothermal systems: Comparing the Ligurian ophiolites with oceanic serpentinites. Geochim. Et Cosmochim. Acta 2012, 91, 283-305. [CrossRef]

9. Alt, J.C.; Shanks, W.C. Sulfur in serpentinized oceanic peridotites: Serpentinization processes and microbial sulfate reduction. J. Geophys. Res. Space Phys. 1998, 103, 9917-9929. [CrossRef]

10. Alt, J.C.; Shanks, W.C. Serpentinization of abyssal peridotites from the MARK area, Mid-Atlantic Ridge: sulfur geochemistry and reaction modeling. Geochim. Et Cosmochim. Acta 2003, 67, 641-653. [CrossRef]

11. Alt, J.C.; Shanks, W.C.; Bach, W.; Paulick, H.; Garrido, C.J.; Beaudoin, G. Hydrothermal alteration and microbial sulfate reduction in peridotite and gabbro exposed by detachment faulting at the Mid-Atlantic Ridge, $15^{\circ} 20^{\prime} \mathrm{N}$ (ODP Leg 209): A sulfur and oxygen isotope study. Geochem. Geophys. Geosystems 2007, 8. [CrossRef]

12. Alt, J.C.; Schwarzenbach, E.M.; Früh-Green, G.L.; Shanks, W.C.; Bernasconi, S.M.; Garrido, C.J.; Crispini, L.; Gaggero, L.; Padron-Navarta, J.A.; Marchesi, C.; et al. The role of serpentinites in cycling of carbon and sulfur: Seafloor serpentinization and subduction metamorphism. Lithos 2013, 178, 40-54. [CrossRef]

13. Bach, W.; Garrido, C.J.; Paulick, H.; Harvey, J.; Rosner, M. Seawater-peridotite interactions: First insights from ODP Leg 209, MAR $15^{\circ}$ N. Geochem. Geophys. Geosystems 2004, 5. [CrossRef]

14. Barnes, J.; Sharp, Z. Achlorine isotope study of DSDP/ODP serpentinized ultramafic rocks: Insights into the serpentinization process. Chem. Geol. 2006, 228, 246-265. [CrossRef]

15. Bonatti, E.; Lawrence, J.R.; Morandi, N. Serpentinization of oceanic peridotites: temperature dependence of mineralogy and boron content. Earth Planet. Sci. Lett. 1984, 70, 88-94. [CrossRef]

16. Boschi, C.; Früh-Green, G.L.; Escartín, J. Occurrence and significance of serpentinite-hosted, talc-and amphibole-rich fault rocks in modern oceanic settings and ophiolite complexes: An overview. Ofioliti 2006, 31, 129-140.

17. Boschi, C.; Dini, A.; Früh-Green, G.L.; Kelley, D.S. Isotopic and element exchange during serpentinization and metasomatism at the Atlantis Massif (MAR $\left.30^{\circ} \mathrm{N}\right)$ : Insights from B and Sr isotope data. Geochim. Et Cosmochim. Acta 2008, 72, 1801-1823. [CrossRef]

18. Delacour, A.; Früh-Green, G.L.; Bernasconi, S.M. Sulfur mineralogy and geochemistry of serpentinites and gabbros of the Atlantis Massif (IODP Site U1309). Geochim. Et Cosmochim. Acta 2008, 72, 5111-5127. [CrossRef] 
19. Delacour, A.; Früh-Green, G.L.; Bernasconi, S.M.; Schaeffer, P.; Kelley, D.S. Carbon geochemistry of serpentinites in the Lost City Hydrothermal System ( $30^{\circ}$ N, MAR). Geochim. Et Cosmochim. Acta 2008, 72, 3681-3702. [CrossRef]

20. Delacour, A.; Früh-Green, G.L.; Bernasconi, S.M.; Kelley, D.S. Sulfur in peridotites and gabbros at Lost City $\left(30^{\circ} \mathrm{N}, \mathrm{MAR}\right)$ : Implications for hydrothermal alteration and microbial activity during serpentinization. Geochim. Et Cosmochim. Acta 2008, 72, 5090-5110. [CrossRef]

21. Früh-Green, G.L.; Connolly, J.A.; Plas, A.; Kelley, D.S.; Grobéty, B.; Wilcock, W.S.; Delong, E.F.; Baross, J.A.; Cary, S.C. Serpentinization of oceanic peridotites: Implications for geochemical cycles and biological activity. Subseafloor Biosph. Mid-Ocean Ridges 2004, 144, 119-136.

22. Kendrick, M.A.; Scambelluri, M.; Honda, M.; Phillips, D. High abundances of noble gas and chlorine delivered to the mantle by serpentinite subduction. Nat. Geosci. 2011, 4, 807-812. [CrossRef]

23. Paulick, H.; Bach, W.; Godard, M.; De Hoog, J.; Suhr, G.; Harvey, J. Geochemistry of abyssal peridotites (Mid-Atlantic Ridge, $15^{\circ} 20^{\prime}$ N, ODP Leg 209): Implications for fluid/rock interaction in slow spreading environments. Chem. Geol. 2006, 234, 179-210. [CrossRef]

24. Scambelluri, M.; Fiebig, J.; Malaspina, N.; Muntener, O.; Pettke, T. Serpentinite Subduction: Implications for Fluid Processes and Trace-Element Recycling. Int. Geol. Rev. 2004, 46, 595-613. [CrossRef]

25. Schwarzenbach, E.M. Serpentinization, fluids and life: Comparing carbon and sulfur cycles in modern and ancient environments. Doctoral dissertation, ETH Zurich, Zurich, Switzerland, 2011; 240p.

26. Vils, F.; Muntener, O.; Kalt, A.; Ludwig, T. Implications of the serpentine phase transition on the behaviour of beryllium and lithium-boron of subducted ultramafic rocks. Geochim. Et Cosmochim. Acta 2011, 75, 1249-1271. [CrossRef]

27. Kelemen, P.B.; Matter, J.; Streit, E.E.; Rudge, J.F.; Curry, W.B.; Blusztajn, J. Rates and Mechanisms of Mineral Carbonation in Peridotite: Natural Processes and Recipes for Enhanced, in situ $\mathrm{CO}_{2}$ Capture and Storage. Annu. Rev. Earth Planet. Sci. 2011, 39, 545-576. [CrossRef]

28. Lacinska, A.M.; Styles, M.T.; Bateman, K.; Hall, M.R.; Brown, P.D. An Experimental Study of the Carbonation of Serpentinite and Partially Serpentinised Peridotites. Front. Earth Sci. 2017, 5, 1-20. [CrossRef]

29. Miller, J.A.; Cartwright, I.; Buick, I.S.; Barnicoat, A.C. An O-isotope profile through the HP-LT Corsican ophiolite, France and its implications for fluid flowduring subduction. Chem. Geol. 2001, 178, 43-69. [CrossRef]

30. Eickmann, B.; Bach, W.; Rosner, M.; Peckmann, J. Geochemical constraints on the modes of carbonate precipitation in peridotites from the Logatchev Hydrothermal Vent Field and Gakkel Ridge. Chem. Geol. 2009, 268, 97-106. [CrossRef]

31. Bach, W.; Rosner, M.; Jöns, N.; Rausch, S.; Robinson, L.F.; Paulick, H.; Erzinger, J. Carbonate veins trace seawater circulation during exhumation and uplift of mantle rock: Results from ODP Leg 209. Earth Planet. Sci. Lett. 2011, 311, 242-252. [CrossRef]

32. Schwarzenbach, E.M.; Früh-Green, G.L.; Bernasconi, S.M.; Alt, J.C.; Plas, A. Serpentinization and carbon sequestration: A study of two ancient peridotite-hosted hydrothermal systems. Chem. Geol. 2013, 351, 115-133. [CrossRef]

33. Cook-Kollars, J.; Bebout, G.E.; Collins, N.C.; Angiboust, S.; Agard, P. Subduction zone metamorphic pathway for deep carbon cycling: I. Evidence from HP/UHP metasedimentary rocks, Italian Alps. Chem. Geol. 2014, 386, 31-48. [CrossRef]

34. Collins, N.C.; Bebout, G.E.; Angiboust, S.; Agard, P.; Scambelluri, M.; Crispini, L.; John, T. Subduction zone metamorphic pathway for deep carbon cycling: II. Evidence from HP/UHP metabasaltic rocks and ophicarbonates. Chem. Geol. 2015, 412, 132-150. [CrossRef]

35. Piccoli, F.; Brovarone, A.V.; Beyssac, O.; Martinez, I.; Ague, J.J.; Chaduteau, C. Carbonation by fluid-rock interactions at high-pressure conditions: Implications for carbon cycling in subduction zones. Earth Planet. Sci. Lett. 2016, 445, 146-159. [CrossRef]

36. De Felipe, I.; Pedreira, D.; Pulgar, J.A.; Iriarte, E.; Mendia, M. Mantle exhumation and metamorphism in the Basque-Cantabrian Basin (N Spain): Stable and clumped isotope analysis in carbonates and comparison with ophicalcites in the North-Pyrenean Zone (Urdach and Lherz). Geochem. Geophys. Geosystems 2017, 18, 631-652. [CrossRef]

37. Structural and petrological analyses of the Frido Unit (southern Italy): New insights into the early tectonic evolution of the southern Apennines-Calabrian Arc system. Lithos 2013, 168, 219-235. 
38. Knott, S.D. Structure, kinematics and metamorphism in the Liguride Complex, southern Apennines, Italy. J. Struct. Geol. 1994, 16, 1107-1120. [CrossRef]

39. Monaco, C.; Tortorici, L. Tectonic role of ophiolite-bearing terranes in the development of the Southern Apennines orogenic belt. Terra Nova 1995, 7, 153-160. [CrossRef]

40. Bonardi, G.; Amore, F.O.; Ciampo, G.; De Capoa, P.; Micconet, P.; Perrone, V. II complesso Liguride. Auct: Stato delle conoscenze e problemi aperti sulla sua evoluzione pre-appenninica ed i suoi rapporti con l'Arco Calabro. Mem. Della Soc. Geol. Ital. 1988, 41, 17-35.

41. Spadea, P. Continental rocks associated with ophiolites in Lucanian Apennine, southern Italy. Ofioliti 1982, 7 , 501-522.

42. Laurita, S.; Prosser, G.; Rizzo, G.; Langone, A.; Tiepolo, M.; Laurita, A. Geochronological study of zircons from continental crust rocks in the Frido Unit (southern Apennines). Acta Diabetol. 2014, 104, 179-203. [CrossRef]

43. Sansone, M.T.C.; Rizzo, G. Rodingites from Frido Unit: Evidences for metasomatic alteration. Rend. Online Della Soc. Geol. Ital. Abstr. 2010, 11, 106-107.

44. Rizzo, G.; Sansone, M.T.C.; Perri, F.; Laurita, S. Mineralogy and petrology of the metasedimentary rocks from the Frido Unit (southern Apennines, Italy). Period. Miner.. 2016, 85, 153-168.

45. Rizzo, G.; Canora, F.; Dichicco, M.C.; Laurita, S.; Sansone, M.T.C. P-T estimates from amphibole and plagioclase pairs in metadolerite dykes of the Frido unit (southern Apennines-Italy) during the ocean-floor metamorphism. J. Mediterr. Earth Sci. 2019, 11. [CrossRef]

46. Sinisi, R.; Mongelli, G.; Perri, F.; Rizzo, G. The braunite $\left(3 \mathrm{Mn}_{2} \mathrm{O}_{3}-\mathrm{MnSiO}_{3}\right)$-rich mineralization in the metasedimentary succession from southern Apennines (Italy): Genesis constraints. Ore Geol. Rev. 2018, 94, 1-11. [CrossRef]

47. Rizzo, G.; Laurita, S.; Altenberger, U. The Timpa delle Murge ophiolitic gabbros, southern Apennines: Insights from petrology and geochemistry and consequences to the geodynamic setting. Period. Miner.. 2018, 87, 5-20.

48. Dichicco, M.C.; Laurita, S.; Paternoster, M.; Rizzo, G.; Sinisi, R.; Mongelli, G. Serpentinite Carbonation for $\mathrm{CO}_{2}$ Sequestration in the Southern Apennines: Preliminary Study. Energy Procedia 2015, 76, 477-486. [CrossRef]

49. Dichicco, M.C.; De Bonis, A.; Mongelli, G.; Rizzo, G.; Sinisi, R. $\mu$-Raman spectroscopy and X-ray diffraction of asbestos' minerals for geo-environmental monitoring: The case of the southern Apennines natural sources. Appl. Clay Sci. 2017, 141, 292-299. [CrossRef]

50. Dichicco, M.C.; Laurita, S.; Sinisi, R.; Battiloro, R.; Rizzo, G. Environmental and Health: The Importance of Tremolite Occurence in the Pollino Geopark (Southern Italy). Geosciences 2018, 8, 98. [CrossRef]

51. Dichicco, M.C.; Castiñeiras, P.; Francisco, C.G.; Acebrón, L.G.; Grassa, F.; Laurita, S.; Paternoster, M.; Rizzo, G.; Sinisi, R.; Mongelli, G. Genesis of carbonate-rich veins in the serpentinites at the Calabria-Lucania boundary (southern Apennines). Rend. Online della Soc. Geol. Ital. 2018, 44, 143-149. [CrossRef]

52. Mazzeo, F.; Zanetti, A.; Aulinas, M.; Petrosino, P.; Arienzo, I.; D’Antonio, M. Evidence for an intra-oceanic affinity of the serpentinized peridotites from the Mt. Pollino ophiolites (Southern Ligurian Tethys): Insights into the peculiar tectonic evolution of the Southern Apennines. Lithos 2017, 284, 367-380. [CrossRef]

53. Sansone, M.T.C.; Rizzo, G.; Mongelli, G. Petrochemical characterization of mafic rocks from Ligurian ophiolites, southern Apennines. Int. Geol. Rev. 2011, 53, 130-156. [CrossRef]

54. Sansone, M.T.C.; Prosser, G.; Rizzo, G.; Tartarotti, P. Spinel peridotites of the Frido unit ophiolites (southern Apennines Italy): Evidence for oceanic evolution. Period. Miner.. 2012, 81, 35-59.

55. Sansone, M.T.C.; Tartarotti, P.; Prosser, G.; Rizzo, G. From ocean to subduction: The polyphase metamorphic evolution of the Frido unit metadolerite dykes (southern Apennine, Italy). J. Virtual Explor. 2012, 41, 3. [CrossRef]

56. Sansone, M.T.C.; Rizzo, G. Pumpellyite veins in the metadolerite of the Frido unit (southern Apennines-Italy). Period. Miner.. 2012, 81, 75-92.

57. Spadea, P. Contributo alla conoscenza dei metabasalti ofiolitici della Calabria settentrionale e centrale e dell'Appennino lucano. Rend. Soc. Ital. Mineral. Petrol. 1979, 35, 251-276.

58. Knott, S.D. The Liguride Complex of Southern Italy-A Cretaceous to Paleogene accretionary wedge. Tectonophys. 1987, 142, 217-226. [CrossRef] 
59. Beccaluva, L.; Maciotta, G.; Spadea, P. Petrology and geodynamic significance of the calabria-lucania ophiolites. Rend. Soc. Ital. Min.. Pet.. 1982, 38, 973-987.

60. Cavalcante, F.; Belviso, C.; Laurita, S.; Prosser, G. P-T constraints from phyllosilicates of the Liguride complex of the Pollino area (southern Apennines, Italy): Geological inferences. Ofioliti 2012, 37, 65-75.

61. Invernizzi, C.; Bigazzi, G.; Corrado, S.; Di Leo, P.; Schiattarella, M.; Zattin, M. New thermobaric constraints of the exhumation history of the Liguride accretionary wedge, Southern Italy. Ofioliti 2008, 33, 21-32.

62. Laurita, S.; Rizzo, G. Blueschist metamorphism of metabasite dykes in the serpentinites of the Frido Unit, Pollino Massif. Rend. Online Della Soc. Geol. Ital. 2018, 45, 129-135. [CrossRef]

63. Cirrincione, R.; Monaco, C. Evoluzione tettonometamorfica dell'Unità del Frido (Appennino Meridionale). Mem. della Soc. Geol. Ital. 1996, 51, 83-92.

64. Laurita, S.; Rizzo, G. The First Occurrence of Asbestiform Magnesio-Riebeckite in Schists in the Frido Unit (Pollino Unesco Global Geopark, Southern Italy). Fibers 2019, 7, 79. [CrossRef]

65. Laurita, S. IL prisma di accrezione liguride affiorante al confine Calabro-Lucano: studio termocronologico e strutturale. Ph.D Thesis, Università degli Studi della Basilicata, Potenza, Italy, 2008; p. 225.

66. Leake, B.E.; Woolley, A.R.; Arps, C.E.S.; Birch, W.D.; Gilbert, M.C.; Grice, J.D.; Hawthorne, F.C.; Kato, A.; Kisch, H.J.; Krivovichev, V.G.; et al. Nomenclature of amphiboles: Report of the Subcommittee on Amphiboles of the International Mineralogical Association, Commission on New Minerals and Mineral Names. Am. Miner. 1997, 82, 1019-1037.

67. Leake, B.E.; Woolley, A.R.; Birch, W.D.; Burke, E.A.J.; Ferraris, G.; Grice, J.D.; Hawthorne, F.C.; Kisch, H.J.; Krivovichev, V.G.; Schumacher, J.C.; et al. Nomenclature of amphiboles: Additions and revisions to the International Mineralogical Association's amphibole nomenclature. Am. Mineral. 2004, 89, 883-887.

68. Coplen, T.B.; Kendall, C.; Hopple, J. Comparison of stable isotope reference samples. Nature 1983, 302, 236-238. [CrossRef]

69. Goldstein, R.H.; Rossi, C. Recrystallization in quartz over-growths. J. Sediment Res. 2002, 72, $432-440$. [CrossRef]

70. Goldstein, R.H. Fluid inclusion geothermometry in sedimentary system: From paleoclimate to hydrothermal. In SEPM Special Publication, Thermal History Analysis of Sedimentary Basins; Harris, N.B., Peters, K.E., Eds.; Society for Sedimentary Geology: Tulsa, OK, USA, 2012; pp. 45-63.

71. Bodnar, R.J. Revised equation and Table for determining the freezing point depression of $\mathrm{H}_{2} \mathrm{O}-\mathrm{NaCl}$ solution. Geochim. Cosmochim. Acta 1993, 57, 683-684. [CrossRef]

72. Whitney, D.L.; Evans, B.W. Abbreviations for names of rock-forming minerals. Am. Miner.. 2010, 95, $185-187$. [CrossRef]

73. Dichicco, M.C.; Paternoster, M.; Rizzo, G.; Sinisi, R. Mineralogical Asbestos Assessment in the Southern Apennines (Italy): A Review. Fibers 2019, 7, 24. [CrossRef]

74. Wicks, F.J.; Whittaker, E.J.W. Serpentine textures and serpentinization. Can. Miner.. 1977, 15, 459-488.

75. Wicks, F.J.; Plant, A.G. Electron-microprobe and X-ray microbeam studies of serpentine textures. Can. Miner. 1979, 17, 785-830.

76. Blaise, S.; Auvray, B. Serpentinization in the Archean komatitic rocks of the kuhmo greenstone belt, eastern Finland. Can. Miner.. 1990, 28, 56-66.

77. Passchier, C.W.; Trouw, R.A.J. Microtectonics; Springer Science \& Business Media: Berlin/Heidelberg, Germany, 2005.

78. Morimoto, N. Nomenclature of pyroxenes. Mineral. Petrol. 1988, 39, 55-76. [CrossRef]

79. Morimoto, N. Nomenclature of pyroxenes. Mineral. J. 1989, 14, 198-221. [CrossRef]

80. Perraki, M.; Proyer, A.; Mposkos, E.; Kaindl, R.; Hoinkes, G. Raman micro-spectroscopy on diamond, graphite and other carbon polymorphs from the ultrahigh-pressure metamorphic Kimi Complex of the Rhodope Metamorphic Province, NE Greece. Earth Planet. Sci. Lett. 2006, 241, 672-685. [CrossRef]

81. O'Neil, J.R. Oxygen Isotope Fractionation in Divalent Metal Carbonates. J. Chem. Phys. 1969, 51, 5547-5558. [CrossRef]

82. Friedman, I.; O'Neil, J.R. Data of Geochemistry: Compilation of Stable Isotope Fractionation Factors of Geochemical Interest; US Government Printing Office: Washington, DC, USA, 1977; Volume 440.

83. Kim, S.T.; O'Neil, J.R. Equilibrium and nonequilibrium oxygen isotope effects in synthetic carbonates. Geochim. Cosmochim. Acta 1997, 61, 3461-3475. [CrossRef] 
84. Schmidt, M.; Xeflide, S.; Botz, R.; Mann, S. Oxygen isotope fractionation during synthesis of CaMg-carbonate and implications for sedimentary dolomite formation. Geochim. Et Cosmochim. Acta 2005, 69, 4665-4674. [CrossRef]

85. Horita, J. Oxygen and carbon isotope fractionation in the system dolomite-water- $\mathrm{CO}_{2}$ to elevated temperatures. Geochim. Et Cosmochim. Acta 2014, 129, 11-124. [CrossRef]

86. Agrinier, P.; Cornen, G.; Beslier, M.O. Mineralogical and oxygen isotopic features of serpentinites recovered from the ocean/continent transition in the Iberia Abyssal Plain. In Proceedings of the ocean drilling program scientific results; National science foundation: Alexandria, VA, USA, 1996; pp. 541-552.

87. Ohmoto, H.; Rye, R.O. Isotopes of sulfur and carbon. Geochemistry of Hydrothermal Ore Deposits; Barnes, H.L., Ed.; John Wiley \& Sons: New York, NY, USA, 1979; pp. 509-567.

88. Roedder, E. Fluid inclusion analysis-prologue and epilogue. Geochim. Et Cosmochim. Acta 1990, 54, 495-507. [CrossRef]

89. Goldstein, R.H.; Reynolds, T.J. Fluid inclusion microthermometry. In Systematics of Ffluid Inclusions in Diagenetic Minerals; Society for Sedimentation Geology: Tulsa, OK, USA, 1994; Short course 31; pp. 87-121.

90. Frost, B.R.; Beard, J.S. On silica activity and serpentinization. J. Petrol. 2007, 48, 1351-1368. [CrossRef]

91. Ghosh, B.; Morishita, T.; Ray, J.; Tamura, A.; Mizukami, T.; Soda, Y.; Ovung, T.N. A new occurrence of titanian (hydro) andradite from the Nagaland ophiolite, India: Implications for element mobility in hydrothermal environments. Chem. Geol. 2017, 457, 47-60. [CrossRef]

92. Moore, D.E.; Rymer, M.J. Talc-bearing serpentinite and the creeping section of the San Andreas fault. Nature 2007, 448, 795. [CrossRef] [PubMed]

93. Klein, C. Mineralogia; Zanichelli: Bologna, Italy, 2004.

94. Simmons, S.F.; Christenson, B.W. Origins of calcite in a boiling geothermal system. Am. J. Sci. 1994, 294, 361-400. [CrossRef]

95. Piccoli, F.; Brovarone, A.V.; Ague, J.J. Field and petrological study of metasomatism and high-pressure carbonation from lawsonite eclogite-facies terrains, Alpine Corsica. Lithos 2018, 304, 16-37. [CrossRef]

96. Scambelluri, M.; Bebout, G.E.; Belmonte, D.; Gilio, M.; Campomenosi, N.; Collins, N.; Crispini, L. Carbonation of subduction-zone serpentinite (high-pressure ophicarbonate; Ligurian Western Alps) and implications for the deep carbon cycling. Earth Planet. Sci. Lett. 2016, 441, 155-166. [CrossRef]

97. Valley, J.W. Stable isotope geochemistry of metamorphic rocks. Rev. Mineral. Geochem. 1986, 16, 445-489.

98. Wang, Q.; Rumble, D. Oxygen and carbon isotope composition from the UHP Shuanghe marbles, Dabie Mountains, China. Sci. China Ser. D Earth Sci. 1999, 42, 88-96. [CrossRef]

99. Ague, J.J.; Nicolescu, S. Carbon dioxide released from subduction zones by fluid mediated reactions. Nat. Geosci. 2014, 7, 355. [CrossRef]

100. Galvez, M.E.; Beyssac, O.; Martinez, I.; Benzerara, K.; Chaduteau, C.; Malvoisin, B.; Malavieille, J. Graphite formation by carbonate reduction during subduction. Nat. Geosci. 2013, 6, 473-477. [CrossRef]

101. Galvez, M.E.; Martinez, I.; Beyssac, O.; Benzerara, K.; Agrinier, P.; Assayag, N. Metasomatism and graphite formation at a lithological interface in Malaspina (Alpine Corsica, France). Contrib. Mineral. Petrol. 2013b, 166, 1687-1708. [CrossRef]

102. Des Marais, D.J.; Moore, J.G. Carbon and its isotopes in mid-oceanic basaltic glasses. Earth Planet. Sci. Lett. 1984, 69, 43-57. [CrossRef]

103. Marty, B.; Jambon, A.; Sano, Y. Helium isotopes and $\mathrm{CO}_{2}$ in volcanic gases of Japan. Chem. Geol. 1989, 76, 25-40. [CrossRef]

104. Sano, Y.; Williams, S.N. Fluxes of mantle and subducted carbon along convergent plate boundaries. Geophys. Res. Lett. 1996, 23, 2749-2752. [CrossRef]

105. Zheng, Y.F. Carbon-oxygen isotopic covariation in hydrothermal calcite during degassing of $\mathrm{CO}_{2}$. Miner. Depos. 1990, 25, 246-250. [CrossRef]

106. Hoefs, J. Stable Isotope Geochemistry; Springer: Berlin/Heidelberg, Germany, 2018; Volume XXVI, p. 437.

(C) 2020 by the authors. Licensee MDPI, Basel, Switzerland. This article is an open access article distributed under the terms and conditions of the Creative Commons Attribution (CC BY) license (http://creativecommons.org/licenses/by/4.0/). 